\title{
CROSS-LINGUISTIC DIFFERENCES IN BILINGUALS' FUNDAMENTAL FREQUENCY RANGES
}

RUNNING TITLE: Pitch range in bilinguals' speech

Authors:

Mikhail Ordin ${ }^{1,2}$

${ }^{1}$ BCBL-Basque Centre on Cognition, Brain and Language

Mikeletegi 69, 20009, Donostia, Spain

${ }^{2}$ IKERBASQUE - Basque Foundation for Science

Maria Diaz de Haro 3, 48013, Bilbao, Spain

$\underline{\text { m.ordin@bcbl.eu }}$

+34943309300 Ext.: 228

Ineke Mennen $^{3}$

${ }^{3}$ University of Graz, Department of English Studies

Heinrichstraße 36/II/327, 8010, Graz, Austria

ineke.mennen@uni-graz.at

$+43(0) 316380-2471$ 


\section{CROSS-LINGUISTIC DIFFERENCES IN BILINGUALS’ FUNDAMENTAL}

\section{FREQUENCY RANGES}

\section{ABSTRACT}

Aims and Objectives: We investigated cross-linguistic differences in fundamental frequency range in Welsh-English bilingual speech.

Methodology/Approach: Fundamental frequency range was conceptualized as a behavioral pattern using measures of span (range of fundamental frequency - in semitones - covered by the speaker's voice) and level (overall height of fundamental frequency maxima, minima, and means of speaker's voice) in each language.

Data and Analysis: Fundamental frequency range measures were taken from recordings of 30 WelshEnglish bilinguals (14 females and 16 males), who read 70 semantically matched sentences, 35 in each language. Comparisons were made within speakers across languages, separately in male and female speech. Language background and language use information was elicited for qualitative analysis of extralinguistic factors that might affect the fundamental frequency range.

Findings: Cross-linguistic differences in fundamental frequency range were found to be consistent across female bilinguals, but random across male bilinguals. Most female bilinguals showed distinct fundamental frequency ranges for each language. Most male bilinguals, however, were found not to change their fundamental frequency range when switching languages. Those who did change employed different strategies than women when differentiating fundamental frequency ranges between languages.

Originality: This is the first study that reports gender-specific behavior in switching fundamental frequency ranges across languages in bilingual speech.

Significance/Implications: Detected cross-linguistic differences in fundamental frequency range can be explained by socio-cultural factors. Consequently, sociolinguistic factors are to be taken into account in any further study of language-specific pitch setting and cross-linguistic differences in fundamental frequency range. 
KEYWORDS: pitch range, fundamental frequency range, F0 span, F0 level, bilingualism, bilingual speech, pitch span, pitch level

\section{INTRODUCTION}

There is ample evidence that spoken languages and dialects may differ in the fundamental frequency range (FFR) characteristically deployed by its speakers (Eady,1982; Dolson, 1994; Chen, 2005; Keating \& Kuo, 2012 among others). A number of possible sources have been posited to account for the observed crosslanguage and cross-varietal differences in FFR. Differences have been attributed to (i) dissimilarities in the intonational structure of linguistic varieties, such as differences in the inventory of boundary tones and pitch accents (Mennen, Schaeffler, \& Docherty, 2012; Yamazawa \& Hollien, 1992); (ii) differences in cultural and social norms of the linguistic communities (Dolson, 1994; van Bezooijen, 1995; Deutsch, Le, Shen, Henthorn, 2009); (iii) ethological factors (Ohala, 1984; Fitch \& Reby, 2001; Lieberman, Klatt, \& Wilson, 1969); and (iv) differences in the anatomy/physiology of speakers (van Dommelen \& Moxness, 1995; Kuenzel, 1989). However, there has been little attempt to distinguish between these possible sources. An investigation of the cross-linguistic use of FFR in bilingual speakers might provide much needed insights into this issue and help determine the underlying source of cross-language differences. First, the use of bilingual speakers will control for anatomical/physiological differences. That is, if the bilingual speakers are found to differ in their two languages, these FFR differences cannot be innately physiological in nature as the comparisons are made within the same subjects. Consequently, by design, this study eliminates one of the potential sources of influence on language-specific FFR. Secondly, the use of bilinguals will also help identify whether FFR is determined by ethological factors. Ohala (1984) proposed that uses of f0 in speech (including variation of FFR) can be explained with reference to an innately specified 'frequency code'. This inherently biological code is based on the correlation between the size of the larynx and the rate of vocal fold vibration, such that smaller larynxes produce higher f0 than larger ones. It is thought that this correlation is universally exploited to express power relations in speech: high pitch sounds submissive and vulnerable, whereas low pitch sounds dominant and aggressive. If FFR were solely determined by such ethological factors we would expect these factors to be similar in the two languages of bilinguals. Thirdly, if 
FFR is determined by the linguistic structure of a certain language, e.g., by language-specific features of intonational phonology, then we should expect to notice the cross-linguistic differences in FFR when bilinguals switch between languages, and the detected change should be noticeable in group comparison as well as in individual comparison of FFR between languages within each speaker, and in both males and females. If, however, FFR is determined by socio-cultural factors, then we might expect to notice that some individuals switch their FFR between languages, while others do not, depending on the extralinguistic factors related to language background.

In the next section we will define the notion of FFR more precisely. We will then review the existing literature on FFR in different populations, and outline the research questions targeted in our study.

\subsection{Fundamental frequency range}

Following Ladd (2008: 188-210), we will define FFR - also referred to as pitch range (Ladd, 2008; Dilley, 2010) and speaking fundamental frequency (Keating \& Kuo, 2012; Awan \& Mueller, 1996) - along two (quasi-independent) dimensions: level and span. Level, or "register" (Cruttenden, 1997) defines the overall height of the speaker's habitual voice. Span, also referred to as "key" (Cruttenden, 1997) or "excursion size" ('t Hart, Collier, \& Cohen, 1990), refers to the range of frequencies covered by an individual when speaking. Span and level - although not entirely independent - can be seen as constituting different aspects of speakers' FFR. That is, two speakers can vary their range of frequencies in a very similar way (showing a very similar or identical span), while one speaker's voice is considerably higher than the other. These speakers vary in their overall level, but have a roughly equal span. Conversely, it is possible for two speakers to have roughly the same height of voice, but to differ in the range of frequencies they cover. These speakers differ in span, but their overall level is roughly similar to each other. Thus, it is worth to distinguish these two aspects of FFR (see also Ladd, 2008; Ladd, Silverman, Tolkmitt, et al., 1985; Cruttenden, 1997; 't Hart, Collier, \& Cohen, 1990 for more evidence of the relative independence of level and span dimensions).

\subsection{Differences in fundamental frequency range between linguistic varieties}


There is growing empirical evidence for the existence of cross-language differences in FFR. Studies indicate that FFR differences are apparent in typologically different languages (e.g. Eady, 1982; Hanley, Snidecor, \& Ringel, 1966; Keating \& Kuo, 2012) as well as in languages within the same language class (e.g. Mennen, Schaeffler \& Docherty, 2012). Furthermore, cross-language differences have been observed in level as well as span dimensions of FFR. For example, when readings of a passage in Japanese, Spanish and American English were compared, Japanese was found to have the highest level, followed by Spanish and then American English (Hanley, Snidecor, \& Ringel, 1966). In later studies, differences in FFR between many other languages have been reported. Amongst others, Polish was found to have a somewhat higher level than American English (Majewski, Hollien \& Zalewski, 1972); Mandarin was found to have a higher level (Eady, 1982; Keating and Kuo, 2012) and wider span compared to English (Keating \& Kuo, 2012; Chen, 2005). Min - another Sinitic language - was found to have a wider span and higher level compared to English (Chen, 2005). Japanese was found to have a higher level than Dutch (van Bezooijen, 1995). Slavic languages were found to have a wider span (Volin, Poesova, \& Weingartova, 2015) and higher level than Germanic languages (Majewski et al., 1972; Holden \& Hogan, 1993; Andreeva, Demenko, Moebius et al., 2014; Volin, et al., 2015). Studies comparing languages belonging to the same language class have received much less attention, but the few existing studies also report differences in FFR. For example, British English females were found to have a wider span (Mennen et al., 2012) and higher level (Mennen et al., 2012; Scharff-Rethfeldt, Miller, \& Mennen, 2008) than German females. Chen (2005), on the other hand, did not find differences in FFR between the two typologically similar languages Mandarin and Min, while each of these languages was significantly different in both FRR dimensions from English.

There is also some evidence that FFR can differ between regional or social varieties of the same language. Deutsch et al. (2009) compared speakers from two phonologically similar dialects of Mandarin and found differences in the level employed in their readings of a short, emotionally neutral article in Standard Mandarin. These findings demonstrate that differences in FFR need not be due to differences in the prosodic structure of language varieties, and the authors argue that they are social or cultural in nature, caused by exposure to the prevailing speech patterns of the linguistic community, as originally proposed in Deutsch (1992). Other studies also indicate that such social or cultural factors might affect FFR of speakers 
sharing the same language. For example, Awan and Mueller (1996) observed differences in FFR even in child speech. Hispanic children in US kindergartens were found to exhibit a higher level than African American and Caucasian American children. Although the authors argue that this is the result of physiology (different racial groups), it is perhaps more plausible to attribute the differences to social/cultural factors given the evidence for a lack of correlation between $\mathrm{f} 0$ and measures of overall height, weight, or body size (Hollien \& Jackson, 1973; Kreiman \& Sidtis, 2011; Kunzel, 1989; Majewski et al., 1972; Rendall, Vokey, \& Nemeth, 2007; van Dommelen \& Moxness, 1995). The plausibility of a link between cultural factors and language-specific FFR is further supported by the finding that exposure to the prevalent speech pattern of a linguistic community not only shapes the FFR of an individual's own speech but even the way they perceive musical patterns (Deutsch, 1992; Dolson, 1994; Ragozzine \& Deutsch, 1994). Further support for an influence of social factors is found by van Bezooijen (1995), who demonstrated that even in languages that are typologically different, FFR differences can be explained by the speakers' desire to conform to sociocultural expectations.

A limited number of studies have compared FFR in first (L1) and second (L2) language speech. Todaka (1990) examined FFR in Japanese speakers who had been learning English for 8-11 years at school in Japan. FFR measures were taken from sentence readings and compared to those obtained from one male and one female native English control speaker. Results showed a narrower span in the Japanese learners of English compared to the native speaker controls. Yamazawa and Hollien (1992) compared FFR in five groups of female speakers: native speakers of Japanese with no knowledge of English; Japanese learners of English who had resided in the US for less than 3 months; Japanese, relatively fluent speakers of English, who had lived for 2 years or more in the US; native American English learners of Japanese who had studied Japanese for more than a year; and monolingual speakers of American English. The results showed that, when averaged over the different language groups, the Japanese women had a higher level than the American females. This confirms earlier reports in the literature that Japanese (particularly in female speakers) has a rather high level (Loveday, 1981; van Bezooijen, 1995). Yamazawa and Hollien (1992) further found a tendency for all Japanese learners of English and American learners of Japanese to distinguish between their two languages, with a higher level in Japanese than in English. Although the 
differences were only marginal, it suggests that the learners may have transferred their native FFR patterns to their L2. Ohara (1999) also compared 10 Japanese learners of American English with 10 American English learners of Japanese. This study, however, aimed to examine the link between cultural factors and language-specific FFR by specifically controlling for the sex of the speaker. Results showed that the pitch level was higher in the Japanese than in the English spoken by the females in both groups of learners. The males, however, did not employ a higher level when speaking Japanese, neither in the group of American learners of Japanese, nor in the Japanese learners of American English. This was interpreted as evidence for a cross-cultural differences in gender-related social meanings conveyed by FFR. However, as the speakers were not bilinguals who had acquired both languages simultaneously (henceforth referred to as simultaneous bilinguals) and therefore were unlikely to be equally proficient in their two languages, transfer of their native FFR patterns to their L2 cannot be ruled out.

Scharff-Rethfeldt, et al. (2008) also found evidence for transfer of FFR in a mixed group of simultaneous and sequential German-English female bilinguals. A different FFR was found for the GermanEnglish females depending on which language they were speaking, with a higher level in their German than in their English. In each language, however, they were found to differ from monolingual speakers. Similar evidence that L1 transfer can have a confounding influence on FFR comes from a study by Mennen, Schaeffler, and Dickie (2014), who investigated whether German learners of English adopted the languageappropriate FFR values of the target language. Their results showed that at those places where span differs most substantially between German and English, the L2 learners of English exhibited spans that were intermediate between those of native German and native English speakers. Although involving L2 learners has the advantage of controlling for physiological differences, the disadvantage is that it introduces the possibility of transfer of FFR from the L1. A further problem when involving L2 learners is the possible influence of anxiety or lack of confidence that L2 learners often experience when speaking in a foreign language. Mennen (1998) showed that Dutch learners of Greek had a narrower span than that of native Greek speakers. Similarly, Volin et al. (2015) found a more compressed span for Czech learners of English in their L2 compared to native English speakers. In both studies, however, span was even more compressed than that of monolingual speakers of the respective languages and can therefore not be the result of L1 
transfer. Both studies argued that these differences in FFR may have resulted from "lack of confidence" (Mennen, 1998: 18) or "anxiety associated with speaking a foreign language" (Volin et al., 2015: 121). This conclusion was also reached in a study by Zimmerer, Jügler, Andreeva, Möbius \& Trouvain (2016: 1037), who speculated that the "clear trend for a compressed pitch range in L2 speech production" could be caused by insecurity and lack of confidence.

Despite the growing interest in cross-language and cross-varietal differences in FFR, the realization of such differences in simultaneous bilinguals received very little attention. Altenberg and Ferrand (2006) investigated FFR in Russian-English bilinguals, Cantonese-English bilinguals, and a monolingual English control group. When speaking in English, no differences were found between the three groups. The RussianEnglish bilinguals, however, were found to have a higher level in their Russian than in their English, thus indicating that some bilinguals have different FFRs for the languages in their repertoire. Cantonese-English bilinguals, on the other hand, did not exhibit distinct FFRs in their different languages. The authors attributed the differences between the bilingual groups to a possible influence of proficiency (the CantoneseEnglish group was less proficient), differences in the prosodic structure of the two language pairs, or to cultural differences. The only other studies with simultaneous bilinguals that we are aware of is a smallscale study by Graham (2014) and Voigt, Jurafsky and Sumnar (2016). Graham (2014) investigated whether 5 male and 5 female Japanese-English bilinguals varied their realization of FFR as a function of the language they were speaking. It was found that both the male and female bilinguals produced Japanese with a wider span and a higher level than English. Graham (2014) argued that these differences in FFR are the result of cross-language differences in prosodic structure, in particular due to differences in the realization of accent peaks. Nevertheless, Graham (2014) did not rule out a possible influence of social or cultural factors, although this was not further explored. The role of social or cultural factors was, however, explored in a recent study by Voigt et al. (2016), who investigated the cross-language realization of FFR in GermanItalian and German-French simultaneous male and female bilinguals. Their results showed that both sets of bilinguals produced different FFR depending on the language they were speaking, and that this effect was strongly modulated by gender. Although their results suggest that social or cultural factors may play an 
important role in FFR, other effects (such as an influence of the length or linguistic structure of the utterances) could not be ruled out as their results were based on spontaneous speech.

In summary, although there is no doubt as to the existence of cross-language and cross-varietal differences in FFR, a number of possible sources have been posited to account for them and no clear attempts have been made to distinguish between sources of influence. Furthermore, as most studies have investigated either male or female speakers, and the sample (ranging from read sentences and texts of various lengths to spontaneous speech) and number of speakers investigated varies dramatically between studies, we are nowhere nearer understanding the causes of cross-language differences in FFR.

In light of this background our study focuses on FFR in the speech of simultaneous Welsh-English bilinguals in an attempt to address the following questions: 1) Do Welsh-English bilinguals exhibit differences in FFR when they use different languages? 2) If so, what dimension(s) of FFR - span or level are language-specific and what strategies are used to differentiate FFR between languages? Also, we will attempt to address the question whether extralinguistic factors might affect FFR in Welsh and English in bilinguals's speech, and if so, what are the potential factors. To approach these questions, we analysed read sentences by 'balanced' Welsh-English bilinguals, who acquired both languages simultaneously from an early age as described shortly, and compared FFR measures in the two languages to detect the overall crosslinguistic differences in male and female groups. We then compared Welsh and English FFR for each speaker individually, to detect whether all bilinguals vary their FFR across their languages and whether this behaviour within individuals is associated with extralinguistic factors related to language use and language background.

\section{METHOD}

\subsection{Participants}

Prior to participation, informed consent was obtained in accordance with a protocol approved by the Institutional Review Board at Bangor University. A total of 32 speakers were recruited for this study, 14 females and 18 males. Their age ranged from 21 to 34, with a median age of 24 . None of the participants had 
any known hearing problems or speech or voice disorders. All participants were childhood bilinguals, who had acquired both languages as infants or in early childhood. Participants were all from North Wales (Gwynedd or Anglesey), to control for possible regional variation in pitch. The speakers were recruited by advertising for those who spoke both Welsh and English routinely since childhood. We only recruited bilinguals who used Welsh and English on a daily basis, in formal (as a medium of education or at work, with colleagues and customers) as well as in informal settings (e.g., at home or with friends). We checked their language use by administering a language background questionnaire (see below). Those bilinguals who reported to use one language only at home, and the other language solely in job environment or in educational settings, were excluded from this study.

As part of the experimental protocol, participants were also asked to complete a languagebackground questionnaire. This was done to elicit the information which language the participants use to talk to their mother, father, which language the parents used to talk to each other, which was the language of social environment (at school or kindergarten) between 0 and 4 years, 4 and 9 years, 9 and 15 years. Also, we were interested in where the person grew up and lived for period longer than 6 months. This information was collected to ensure that our participants were balanced bilinguals and had grown up in a bilingual environment.

\subsection{Speech material, procedure, and recordings}

We selected a scholarly text on the history of the Welsh language (in Welsh) and a similar text on the history of the English language (in English). We also used two sets of 47 semantically equivalent colloquial sentences, one for each language. Each speaker was randomly assigned to one of two groups. Half of the participants were asked to read the Welsh text out loud, followed by the reading of the set of 47 colloquial sentences in Welsh. They then read the English material (text followed by sentences) out loud. The other half of the participants first read the English material (text followed by sentences) out loud, followed by the reading of the Welsh material. Only 35 sentences per speaker per language were used for subsequent acoustic analysis, as we discarded the first 10 and the last 2 sentences of each recording. This was done to 
avoid effects of list reading, and remove a possible lowering of $\mathrm{f} 0$ at the end of a list of sentences and a possible readjustment of the pitch range when switching language.

Recordings were made in a sound-proof room at Bangor university or in a quiet room $^{1}$, using a condenser microphone attached to a Marantz solid state recorded, at 44kHz sampling frequency, at 16 bit, in PCI format, mono. In all cases, the microphone was placed on a table directly in front of the participants at a distance of approximately 20 centimeters and keeping an approximate 45 degree microphone-to-mouth angle. Although recordings were made of 14 female and 18 male adult Welsh English bilingual participants, the recordings of two male participants (male speakers 2 and 18) were excluded from subsequent analysis. The data of speaker 2 were discarded because he had a very hesitant reading style, with many hesitation pauses, false starts, intermediate phrase breaks and reading errors (while reading the text). Recordings of speaker 18 were excluded for technical reasons. The recording was contaminated with noise that would interfere with measures of FFR. Thus, we retained 14 female and 16 male participants for analysis.

\subsection{Acoustic and statistical analysis}

We used three measures to capture the differences in level: F0 maximum, F0 minimum, and mean F0 of each sentence. F0 maxima and minima were identified and annotated manually in Praat (Broersma, 2001) and care was taken to avoid areas of creaky voice or clear F0 tracking errors (such as octave errors). Mean F0 values on each sentence were calculated using the Praat-internal autocorrelation algorithm with $0.01 \mathrm{sec}$ time step and default settings for voicing threshold. For span, we computed the difference between maximum and minimum F0 over each sentence. The resulting Hertz value was then expressed in semitones (ST).

For statistical analysis, we applied Wilcoxon signed rank tests because some data samples of speaker*language combinations revealed deviations from normality (based on Kolmogorov-Smirnov test). The use of non-parametric statistics also complied with the common recommendations and accepted practice to apply non-parametric tests to study cross-linguistic differences in pitch range within individuals (e.g.,

\footnotetext{
${ }^{1}$ This was done for practical reasons, as the recording booth was in the process of being installed when we started the experiment, and we were not able to delay our study. Upon availability of the booth, we recorded all remaining participants there. We made sure that there was an equal distribution of males and females recorded in the booth or quiet room (6 males and 5 females in the sound-treated room, 10 males and 9 females in the booth), and that the location did not affect the results.
} 
Altenberg \& Ferrand, 2006; Mennen et al., 2012). FFR measures were introduced into the tests as dependent variables, and the language as an independent variable. For stringency of analysis, we introduced the HolmBonferroni stepwise correction for multiple comparisons and potential alpha inflation, following the method described and recommended for health-related research in Aickin and Gensler (1996). In the rest of the manuscript, we report the corrected $p$-values, keeping the overall alpha level at 0.05 .

\section{RESULTS}

\subsection{Overall results}

The three measures of level and the measure of span were averaged across all sentences for each speaker to estimate the overall cross-linguistic differences in FFR in bilingual speech, separately in female (Figure 1) and male (Figure 2) speech productions. The overall averaged results are presented in Table 1. The results for the female speakers show a significant effect for span (as measured by the difference between maximum and minimum F0), with a wider span (in ST) exploited in the bilinguals' Welsh than in their English, $z=-$ $3.37, p=.006, r=.64$.

Of the three level measures that were tested, only F0 maximum was found to differ significantly between Welsh and English. That is, F0 maxima were found to be $1.36 \mathrm{ST}$ on average higher in the bilingual females' Welsh than in their English, $z=-3.24, p=.004, r=.61$. The second level measure, F0 minimum, was not significantly different between Welsh and English, $z=-2.17, p=.06, r=.41$, amounting to a small difference of 0.2 ST (or 0.03 Bark) or $1.3 \%(166.4 \mathrm{~Hz}$ in English and $164.2 \mathrm{~Hz}$ in Welsh). The third level measure, F0 mean, was also not significantly different between the Welsh and English employed by the female bilingual speakers, $\mathrm{z}=-1.849, \mathrm{p}=.064, \mathrm{r}=.35$, amounting to a difference of just $0.5 \mathrm{ST}$ or 0.07 Barks, which is $2.76 \%(217 \mathrm{~Hz}$ in Welsh and $211.5 \mathrm{~Hz}$ in English).

As for the males, our analysis did not reveal significant cross-linguistic differences either for the span measure, $z=-1.241, p=0.86, r=0.22$, or for the various level measures (F0 maxima: $z=-.827, p=$ $.408, r=.15$; F0 minima: $z=-.672, p=.501, r=.12$; mean F0: $z=-.414, p=.679, r=.07)$.

\section{TABLE 1 SHOULD BE SOMEWHERE HERE}


FIGURE 1 SHOULD BE SOMEWHERE HERE

FIGURE 2 SHOULD BE SOMEWHERE HERE

In a subsequent analysis, we explored whether the detected trend for a wider span and higher F0 maxima is also observed in individual bilingual speakers.

\subsection{Individual results}

We refer to Table 2 for the statistical results for all speakers. The analysis of the data from each individual bilingual showed that the detected trend is consistent for most female speakers, and is only observed in the speech of a few male bilinguals. A closer look at Table 2 and Figure 3 shows that span in Welsh is significantly wider than in English in the productions of 10 out of 14 female speakers. In the productions of the other four female bilinguals - speakers 2, 3, 6 and 12 - span in Welsh is also wider compared to English, but this difference is not significant. F0 maxima are also found to be higher in Welsh compared to English in the speech of 10 out of 14 female bilinguals. Only four speakers - speakers 2, 6, 12 and 14 - do not reveal significantly higher F0 maxima in Welsh. Interestingly, these include the three speakers (speakers 2, 6, and 12) that also do not have a wider span when speaking Welsh. Individual Welsh and English FFR in female bilinguals' speech does not differ in terms of F0 minima, with the exception of speaker 11, who has slightly (0.4ST) higher F0 minima in English than in Welsh. Mean F0 differs cross-linguistically in the speech of 7 bilingual females: 6 have a higher mean pitch in Welsh, and 1 (speaker 9) reveals a higher mean pitch in English. As F0 minima values do not reveal cross-linguistic differences, we believe that the differences in mean F0 are the consequences of higher F0 maxima and a wider F0 span in Welsh compared to those in English. Bilingual female speakers therefore enhance their span by increasing the F0 peaks, and the random differences in F0 means and minima result from significant and substantial differences in F0 maxima.

\section{FIGURE 3 (a-d) SHOULD BE SOMEWHERE HERE}

The cross-linguistic differences in FFR in male bilingual speech (Table 2, Figure 4) are considerably less pronounced than those in females. Only half of 16 male speakers manifest different FFR in Welsh and English, i.e. speakers 3, 5, 6, 7, 9, 10, 16, 17. Moreover, even these individual exhibit inconsistent patterns regarding which language - Welsh or English - is produced with a wider span or higher level. Male 
speakers 9, 16 and 17 employ a wider span and higher level (raising F0 maxima in all three speakers, F0 minima in speaker 9, and mean F0 in speakers 9 and 16) in Welsh than in English. Male speaker 7 produces Welsh sentences with a higher level, raising both F0 maxima and minima, while male speaker 10 has slightly higher F0 minima in Welsh compared to English. Speaker 5 reveals a contrary tendency: he employs a higher level (higher maxima, mean and minima F0) and wider span in English compared to Welsh. Also, we observed that speaker 6 produces English sentences with a higher mean F0, and also has a wider span and higher F0 maxima in English (although this difference is only marginal). Finally, speaker 3 uses a higher mean and F0 and F0 minima in English.

FIGURE 4 (a-d) SHOULD BE SOMEWHERE HERE

\section{DISCUSSION}

The results indicate that only the speech of the female bilinguals in this study reveals cross-linguistic differences in FFR, and that this is evident in both dimensions: span and level. The bilingual females employed a wider span and higher F0 maxima in Welsh than in English. No differences were found in FFR between Welsh and English produced by the male bilingual speakers in our study. However, FFR differences were observed in the female bilingual speakers, with a wider span and a higher level in their Welsh than in their English.

The experimental evidence presented in this paper lends plausibility to the assumption that the underlying source of the detected cross-language difference in FFR is sociocultural rather than linguistic in nature. The use of childhood bilingual speakers as the participants in this study eliminated one of the potential sources of influence on language-specific FFR, i.e. an influence of physiological differences between speaker groups. As comparisons were made within the same subjects, an influence of physiology on the detected cross-language FFR differences in Welsh and English can be ruled out. Our evidence also makes it unlikely that the underlying source of the detected differences in FFR is linguistic dissimilarities between the two languages. Had the observed difference in FFR been caused by phonological factors - e.g. cross-linguistic differences in the realization or distribution of pitch accents or other dissimilarities in intonational structure between Welsh and English - then we should observe this in both male and female speech. However, that is not what we found. While differences in FFR between Welsh and English are 
evident in the female speakers, the male speakers do not differentiate FFR in their two languages. It would be difficult to explain these differential findings for males and females as the result of intonational dissimilarities between the languages. While there may well be differences between the intonational systems of Welsh and English - for example, in our data we noticed that pitch accents in the English materials were more often falls or high level pitch accents, while in the Welsh materials rises were more commonly used (see also Williams, 1985; Ball, 1989; Cooper, 2015) - one would expect any such influence to be evident in both males and females.

Further support for a sociocultural explanation of the detected cross-language differences in FFR comes from the variation observed in individual bilingual speakers. If the detected cross-language difference in FFR were a function of the phonological/intonational structure of the language spoken, we would expect them to be realized by all speakers. Yet, although the female speakers in our study show a very consistent pattern, there is considerable variation between the male speakers. Most female bilinguals employ different FFR in their languages, and the differences are in the same direction: Welsh speech exhibits a wider span and higher F0 maxima than English. Males are less consistent: some exhibit a higher F0 in English (e.g., see the data in Table 2 for male speakers 3, 5 and 6), some feature a higher level and wider span in Welsh (e.g., see the data in Table 2 for male speakers $7,9,16,17)$, but the majority of male bilinguals do not employ different FFR in their two languages. It would be difficult to attribute such individual differences across speakers to underlying dissimilarities in intonational phonology between Welsh and English, as these should be the same for all speakers.

Also, when inspecting the behavior of the speakers that do differentiate FFR in their two languages, (i.e., most bilingual females and a few males), it can be seen that females and males use different strategies to manifest differences in FFR. Females adjust F0 maxima and leave F0 minima intact. Their mean F0 is also not affected. Thus the cross-linguistic differences in FFR are expressed in span and F0 maxima in female speech. Men, on the other hand, tend to adjust the dimension of level. Those male speakers who have distinct FFR for Welsh and English, tend to increase F0 minima and maxima, or have a higher mean F0 in one of the languages. Differences in level do not always lead to span differences. Raising both F0 maxima and minima may compensate for each other and cancel span widening. The observed individual cases of F0 
span widening in one of the languages (i.e., speakers 5, 9, 16, 17) are accompanied with significant crosslinguistic differences in pitch level, and the effect sizes for the level differences are bigger than those for span differences. On the other hand, some individual cases characterized by significant cross-linguistic level differences are not accompanied with span widening (i.e., male speakers 3, 6, 7 10). Such a gender-specific strategy is also difficult to explain as resulting from structural dissimilarities between Welsh and English.

In order to explore the possible socio-cultural causes of FFR differences in male speech (i.e., to understand why some males differentiate FFR in their two languages, while others do not, and why some males exhibit higher F0 in Welsh, while some prefer to produce higher F0 in English), we had a closer look at the responses in the language-background questionnaire.

We concentrated on the questions related to the use of language (language of exposure - language used as a medium of education, and home language - language used to talk to each of the parents and language used by parents to talk to each other) ${ }^{2}$. The data from the questionnaires is summarized in Tables 3 and 4. Qualitative analysis shows that males 1, 3, 5, 6, and 12 had been exposed to English more than to Welsh, and have English as their home language. Of these speakers, speakers 3, 5, 6 and 12 have a significantly higher F0 in English than in Welsh (speaker 5 exhibits differences in all level parameters, speaker 3 differentiates mean and minima F0, speaker 12 has different minima characteristics in FFR, and speaker 6 exhibits different means). Also, a wider span in English is observed in utterances produced by speakers 5 and 6 (although only marginally significant for speaker 6). Only one participant from the group of speakers with English as their home language, namely, participant 1, does not exhibit cross-language differences in FFR characteristics. In contrast, none of the 11 bilinguals whose home language or language of exposure in childhood was (mostly) Welsh exhibited a higher F0 in English, but some of them (i.e., 7, 9, 16, 17) had significantly higher values of one or several level parameters in Welsh compared to English. This indicates that the language parents use to talk to their child and to communicate with each other, as well

\footnotetext{
${ }^{2}$ In North Wales compulsory education starts at the age of five. The questionnaire shows three questions related to the language of exposure at different ages. Language of exposure between 0 and 4 years is supposed to reflect the language used in the kindergarten (not compulsory) or in the community. Language of exposure between 4 and 9 years is supposed to reflect the language of the primary school, which is usually Welsh, except for some cases where a person used to go to a neighboring village across the border of Gwynned in order to attend an English-speaking primary school. Language of exposure between 9 and 15 years is supposed to reflect the medium of education at secondary school. In North Wales children have an option to attend either a Welsh-medium of English-medium school.
} 
as the language of the environment (e.g., language used as a medium of education) may indeed influence the emergence of cross-linguistic differences in FFR, at least in the speech of the male bilingual participants in our study.

Female bilinguals, on the other hand, tend to consistently switch FFR between languages, and this tendency cannot be explained by differences in language use at home or in the environment. Even those female bilinguals who had English as a medium of education (speaker 5), or used English as a home language (e.g., speakers 4, 5, 11, 13, etc.) have higher and wider FFR in Welsh than in English. This can lead us to one of two possible conclusions: either home language is an influential factor leading to the emergence FFR cross-language differences only in male bilingual population, or we need to explore other possible factors that affect FFR in bilingual speech. It is possible that the extralinguistic factors affecting FFR are altogether different for males and females, and language use may or may not be one of these factors.

It is well-known that the language we speak interacts with cultural and personal identity and modulates speech characteristics and processing (Danziger \& Ward, 2010; Ogunnaike, Dunham, \& Banaji, 2010; Ellis, Kuipers, et al., 2015; Briley, Morris, \& Simonson, 2005). Identity, or personhood, is a decisive cultural characteristic. We can speculate that males need to manifest their individuality, while females tend to conform to the society norms, and that this may have influenced the gender differences in the realization of cross-language differences in FFR. It has often been proposed that men are raised to be more competitive and independent, while women are raised to be more cooperative (Bussey and Bandura, 1999; Eagly, 1978; Huston, 1983; Zahn-Waxier, Cole, \& Barrett, 1991). Research suggests that women tend to accommodate to the societal expectations and to agree with the interlocutors more than men in order to ensure positive social relationships (Eagly, 1978; Cacioppo \& Petty, 1980). Men, on the other hand, are more likely to show nonconformity, especially when this allows them to gain and express a higher level of independence (Griskevicius, Goldstein, Mortensen, Cialdini \& Kenrick, 2006). This difference in the need to conform to societal norms is thought to extend to male and female speech patterns, such that "male speakers are socialized into a competitive style of discourse, while women are socialized into a more cooperative style of 


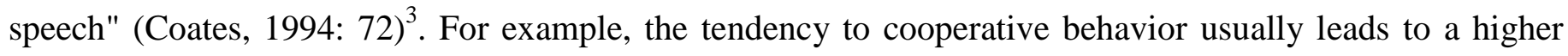
degree of accommodation, i.e., adjusting speech patterns to the patterns of the other members of social interaction (Giles, Tylor, \& Bourhis, 1973; Babel, 2010). As accommodation reduces the social distance and enhances cooperative communication, females in general tend to accommodate more than males (Namy, Lynne, \& Denise, 2002). These differences may well explain the different behavior in the realization of FFR between the males and females in our study. Where women conform to the values that are prevalent in each language, men tend to conform less, and assert their independence by not changing the FFR characteristics when switching languages.

In short, we cannot attribute the cross-language differences in FFR to anatomical differences because the detected changes were observed within individuals. Also, our evidence suggests that these differences are unlikely to be attributed to structural dissimilarities between languages. Besides, we have a clear genderspecific difference in strategies and consistency of switching FFR across languages. Therefore, we can suggest that switching the FFR characteristics across languages is a learnt behavioral pattern, and male and female speakers manifest behavior that is more appropriate to what has historically been considered typical gender expressions. Appropriateness of behavior is determined by sociocultural factors, by societal expectations. We suggest that sociocultural factors should be considered in further studies on FFR to explain phonetic differences in speaker-specific settings for voice profiles in different languages. The exact mechanisms and sociocultural factors that trigger the changes in FFR need further investigation, and our proposed interpretations of the obtained results are preliminary. However, evidence from other studies, correlations and details that we have found in our samples encourage taking a more in-depth look in further studies at gender-specific strategies to express cross-linguistic differences in FFR, and at factors which might trigger these changes in male and female populations.

Our results may also have potential clinical implications. Measures of FFR, particularly those related to the level of a speaker's habitual voice, are considered important parameters in the assessment and treatment of voice disorders (e.g. Scharff-Rethfeldt, et al., 2008; Roy \& Hendarto, 2005). FFR measures are

\footnotetext{
${ }^{3}$ Although there is support for the view that women tend to be less competitive conversationalists compared to men, this gender difference is also influenced by the conversational setting and speech community and may therefore not always be evident.
} 
compared with normative data to establish whether a client's voice quality is pathological and intervention is required. However, what's considered the norm in one language may be considered pathological in another (Scharff-Rethfeldt, et al., 2008). For bilinguals, it is therefore important to consider both languages and compare each with the relevant norms. Our results suggest that this may be particularly important in female bilinguals, as they may be more likely than males to express cross-language differences in FFR. It may also be important to consider cross-language differences in FFR in the treatment of transgender voice. One of the most common targets in the treatment of transgender voice, particularly when treating individuals with male-to-female gender transitions, is that of FFR (Hancock \& Garabedian, 2013). Therapy aims to elevate the FFR of male-to-female individuals in order to reach certain f0 levels that are perceived as female (Hancock \& Garabedian, 2013). However, what is perceived as male or female may differ depending on the language background of the client and the socio-cultural expectation of their linguistic community (Mennen et al., 2012; Voigt et al., 2016). In order to provide appropriate assessment and treatment, normative data are needed for male and female speakers, and for bilingual and multilingual communities with different combinations of languages and cultures. Further research is needed to establish the extent to which FFR is used by male and female speakers to mediate gender and linguistic community (Voigt, et al., 2016), and how speakers are perceived who have FFR values that do not conform to the gender and/or community norms (Mennen et al., 2012).

\section{REFERENCES}

Aickin, M., \& Gensler, H. (1996). Adjusting for multiple testing when reporting research Results: The Bonferroni vs. Holm Methods. American Journal of Public Health 86(5), 726-728.

Altenberg, E. P., \& Ferrand, C. T. (2006). Fundamental frequency in monolingual English, bilingual EnglishRussian, and bilingual English-Cantonese young adult women. Journal of Voice 20, 89-96.

Andreeva, B., Demenko, G., Möbius, B. Zimmerer, F., Jügler, J., \& Jastrzebska, M. (2014). Differences of Pitch Profiles in Germanic and Slavic Languages. In: Proceedings of Interspeech 2014, Singapore: ISCA.

Awan, S. N., \& Mueller, P. B. (1996). Speaking fundamental frequency characteristics of white, African American, and Hispanic kindergartners. J. Speech. Hear. Res. 39, 573-577. 
Babel, M. (2010). Dialect convergence and divergence in New Zealand English. Language in Society 39 (4), 437456.

Baken, R., \& Orlikoff, R. (2000). Clinical Measurement of Speech and Voice. 2nd Ed. San Diego: Singular Publishing.

Ball, M. (1989). The transcription of suprasegmentals in Welsh. Journal of the International Phonetic Association 19, 89-96.

Barsties, B., Verfaillie, R., Dicks, P., \& Maryn, Y. (2014). Is the speaking fundamental frequency in females related to body height? Logopaedics, Phoniatrics, Vocology. Early Online: 1-6 [Epub ahead of print]. Doi: 10.3109/14015439.2014.941928.

Briley, D. A., Morris, M. W., \& Simonson, I. (2005). Cultural chameleons: Biculturals, conformity motives, and decision making. Journal of Consumer Psychology 15, 351-362.

Broersma, P. (2001). Praat, a system for doing phonetics by computer. Glot International 5:9/10, 341-345.

Bussey, K., \& Bandura, A. (1999). Social cognitive theory of gender development differentiation. Psychological Review 106, 676-713.

Cacioppo, J., \& Petty, R. (1980). Sex differences in influenceability: Towards specifying the underlying processes. Personality and Social Psychology Bulletin 6(4), 651-656.

Chen, S. (2005). The effects of tones on speaking fundamental frequency and intensity ranges in Mandarin and Min dialects. J. Acoust. Soc. Am. 117, 3225-3230.

Coates, J. (1994). The language of the professions: Discourse and Career. In J. Evetts (Ed.). Women and Career: Themes and Issues in advanced industrial societies (pp. 72-86). London: Longman.

Cooper, S. (2015). Intonation in Anglesey Welsh. $\mathrm{PhD}$ thesis, Prifysgol Bangor University. (http://e.bangor.ac.uk/4740/1/Cooper\%202015\%20Intonation\%20in\%20Anglesey\%20Welsh.pdf, accessed on 13.03.2016)

Cruttenden, A. (1997). Intonation. $2^{\text {nd }}$ edition. Cambridge: CUP.

Danziger, S. \& Ward, R. (2010). Language changes implicit associations between ethnic groups and evaluation in bilinguals. Psychological Science, 21, 799-800. 
Deutsch, D. (1992). Some new pitch paradoxes and their implications,” Philos. Trans. R. Soc. London, Ser. B. 336, 391-397

Deutsch, D., Le, J., Shen, J., \& Henthorn, T. (2009). The pitch levels of female speech in two Chinese villages. $J$. Acoust. Soc. Am. 125, EL208-EL213.

Dilley, L. (2010). Pitch range variation in English tonal contrasts: Continuous or categorical? Phonetica 67, 63-81.

Dolson, M. (1994). The pitch of speech as a function of linguistic community. Music Perception 11, 321-331.

Eady, S. J. (1982). Differences in the F0 patterns of speech: Tone language versus stress language. Language and Speech 25, 29-42.

Eagly, A. H. (1978). Sex differences in influenceability. Psychological Bulletin, 85, 86-116.

Ellis, C., Kuiper, J., Thierry, G., Lovett, V., Turnbull, O., \& Jones, M. (2015). Language and culture modulate online semantic processing. Social Cognitive and Affective Neuroscience 10, 1392-11396.

Fastl, H., \& Zwicker, E. (2007). Psychoacoustics: Facts and Models. Berlin: Springer.

Fitch, T. \& Reby, D. (2001), The descended larynx is not uniquely human. Proceedings of the Royal Society, B, 268(1477), 1669-1675.

Giles, H., Taylor, D. M., \& Bourhis, R. Y. (1973). Towards a theory of interpersonal accommodation through language: Some Canadian data. Language in Society 2, 177-192.

Graddol, D., \& Swann. J. (1983). Speaking fundamental frequency: Some physical and social correlates. Language and Speech 26, 351-366.

Graham, C. (2014). Fundamental frequency range in Japanese and English: The case of simultaneous bilinguals. Phonetica 71, 271-295.

Griskevicius, V., Goldstein, N. J., Mortensen, C. R., Cialdini, R. B., \& Kenrick, D. T. (2006). Going along versus going alone: When fundamental motives facilitate strategic (non)conformity. Journal of Personality and Social Psychology, 91, 281-294.

Hancock, A.B. \& Garabedian, L. (2013). Transgender voice and communication treatment: a retrospective chart review of 25 cases. International Journal of Language and Communication Disorders 48 (1), 54-65. 
Hanley, T. D., Snidecor, J. C., \& Ringel, R. (1966). Some acoustic differences among languages. Phonetica 14, 97 $-107$.

Holden, K., \& Hogan, J. (1993). The emotive impact of foreign intonation: An experiment in switching English and Russian intonation. Language and Speech 36(1), 67-88.

Hollien, H., \& Jackson, B. (1973). Normative data on the speaking fundamental frequency characteristics of young adult males. Journal of Phonetics 1, 117-120.

Huston, A. C. (1983). Sex typing. In P. H. Mussen (Series Ed.) \& E. M. Hetherington (Vol. Ed.), Handbook of Child Psychology: Vol. 4. Socialization, Personality, and Social Development (4th ed., pp. 387-467). New York: Wiley.

Keating, P., \& Kuo, G. (2012). Comparison of speaking fundamental frequency in English and Mandarin. $J$. Acoust. Soc. Am. 132, 1050-1060.

Kreiman, J., \& Sidtis, D. (2011). Foundations of Voice Studies: An Interdisciplinary Approach to Voice Production and Perception. Oxford: Wiley and Sons.

Kuenzel, H. (1989). How does fundamental frequency correlate with speaker height and weight? Phonetica 46(13), 117-125.

Ladd, R. (2008). Intonational Phonology. $2^{\text {nd }}$ edition. Cambridge: CUP.

Ladd, R., Silverman, K., Tolkmitt, F., Bergmann, G. \& Scherer, K. (1985). Evidence for the independent function of intonation contour type, voice quality, and F0 range in signalling speaker affect. J. Acoust. Soc. Am.78(2), 435444.

Liebermann, P., Klatt, D., \& Wilson, W. (1969). Vocal tract limitations on the vowel repertoires of rhesus monkey and other nonhuman primates. Science 164, 1185-1187.

Loveday, L. (1981). Pitch, politeness and sexual role: An exploratory investigation into the pitch correlates of English and Japanese politeness formulae. Language and Speech 24, 71-89.

Majewski, W., Hollien, H. , \& Zalewski, J. (1972). Speaking fundamental frequency of Polish adult males . Phonetica 25, $119-125$ 
Mennen, I. (1998). Can language learners ever acquire the intonation of a second language? Proceedings of the ESCA Workshop on Speech Technology in Language Learning (pp. 17-20). Stockholm.

Mennen, I., Schaeffler, F., \& Dickie, C. (2014). Second language acquisition of pitch in German learners of English. Studies in Second Language Acquisition 36, 303-329.

Mennen, I., Schaeffler, F., \& Docherty, G. (2012). Cross-language differences in fundamental frequency range: A comparison of English and German. J. Acoust. Soc. Am. 131, 2249-2260.

Namy, L., Lynne, N., \& Denise, S. (2002). Gender Differences in Vocal Accommodation: The Role of Perception. Journal of Language and Social Psychology 21, 422-32.

Ogunnaike, O., Dunham, Y., \& Banaji, M. R. (2010). The language of implicit preferences. Journal of Experimental Social Psychology, 46, 999-1003.

Ohala, J. (1984). An ethological perspective on common cross-language utilization of F0 of voice. Phonetica 41, $1-16$.

Ohara, Y. (1999). Performing gender through voice pitch: A cross-cultural analysis of Japanese and American English. In: U. Pasero \& F. Broun (Eds.). Wahrnehmung und Herstellung von Geschlecht. Wiesbaden: Westdeutscher Verlag.

Ragozzine, F. \& Deutsch, D. (1994). A regional difference in perception of the tritone paradox within the United States. Music Perception 12, 213-225.

Rendall, D., Vokey, J. R., \& Nemeth, C. (2007). Lifting the curtain on the Wizard of Oz: Biased voice-based impressions of speaker size. J. Exp. Psychol. Hum. Percept. Perform. 33, 1208-1219.

Roy, N. \& Hendarto, H. (2005). Revisiting the pitch controversy: changes in speaking fundamental frequency (SFF) after management of functional dysphonia. Journal of Voice 19(4), 582-591.

Scharff-Rethfeldt, W., Miller, N., \& Mennen, I. (2008). Unterschiede in der mittleren Sprechtonhöhe bei Deutsch/Englisch bilingualen Sprechern. Sprache, Stimme, Gehör 32, 123-128.

t' Hart, J., Collier, R., \& Cohen, A. (1990). A perceptual Study of Intonation. Cambridge: CUP.

Todaka, Y. (1990). An Error Analysis of Japanese Students' Intonation and Its Pedagogical Applications. MA Thesis, University of California, Los Angeles. 
Van Bezooijen, R. (1995). Sociocultural aspects of pitch differences between Japanese and Dutch women. Language and Speech 38, 253-265.

Van Dommelen, W. A., \& Moxness, B. H. (1995). Acoustic parameters in speaker height and weight identification: Sex-specific behavior. Language and Speech 38, 267-287.

Voigt. R., Jurafsky, D., \& Sumner, M. (2016). Between- and within-speaker effect of bilingualim on F0 variation. Interspeech 2016, 1122-1126.

Volin, J., Poesova, K., \& Weingartova, L. (2015). Speech melody properties in English, Czech and Czech English: Reference and interference. Research in Language 13(1), 107-123.

Williams, B. (1985). Pitch and duration in Welsh stress perception: the implications for intonation. Journal of Phonetics 13, 381-406.

Yamazawa, H., \& Hollien, H. (1992). Speaking fundamental frequency patterns of Japanese Women. Phonetica $49,128-140$.

Zahn-Waxler, C., Cole, P. M., \& Barrett, K. C. (1991). Guilt and empathy: Sex differences and implications for the development of depression. In J. Garber \& K. A. Dodge (Eds.), The Development of Emotion Regulation and Dysregulation (pp. 243-272). New York: Cambridge University Press.

Zimmerer, F., Jügler, J., Andreeva, B., Möbius, B. \& Trouvain, J. (2014). Too cautious to vary more? A comparison of pitch variation in native and non-native productions of French and German speakers. Proceedings of Speech Prosody 7, Dublin: Trinity College, 1037-1041. 
Figure 1. Cross-linguistic differences in FFR between Welsh and English in female bilingual speech. Error bars stand for $\pm 2 \mathrm{SE}$
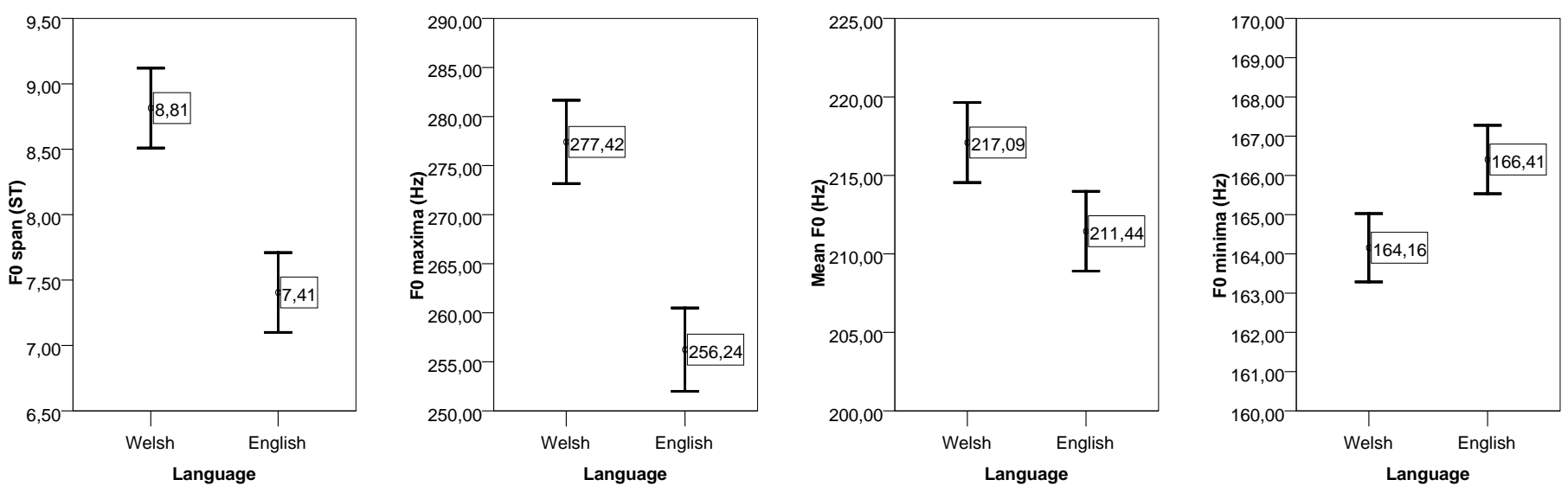
Figure 2. Cross-linguistic differences in FFR between Welsh and English in male bilinguals' speech. Error bars stand for $\pm 2 \mathrm{SE}$
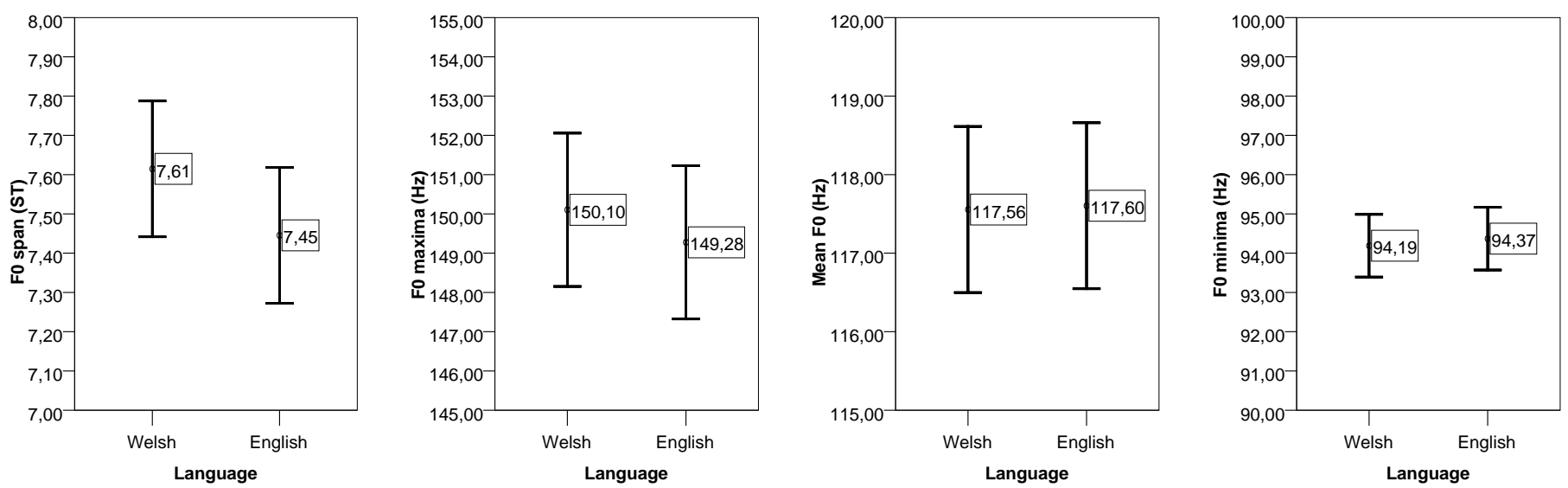
Figure 3a. Individual differences between Welsh and English in span in female bilinguals. Numbers on the graphs stand for the participant's number. Error bars $\pm 2 \mathrm{SE}$.

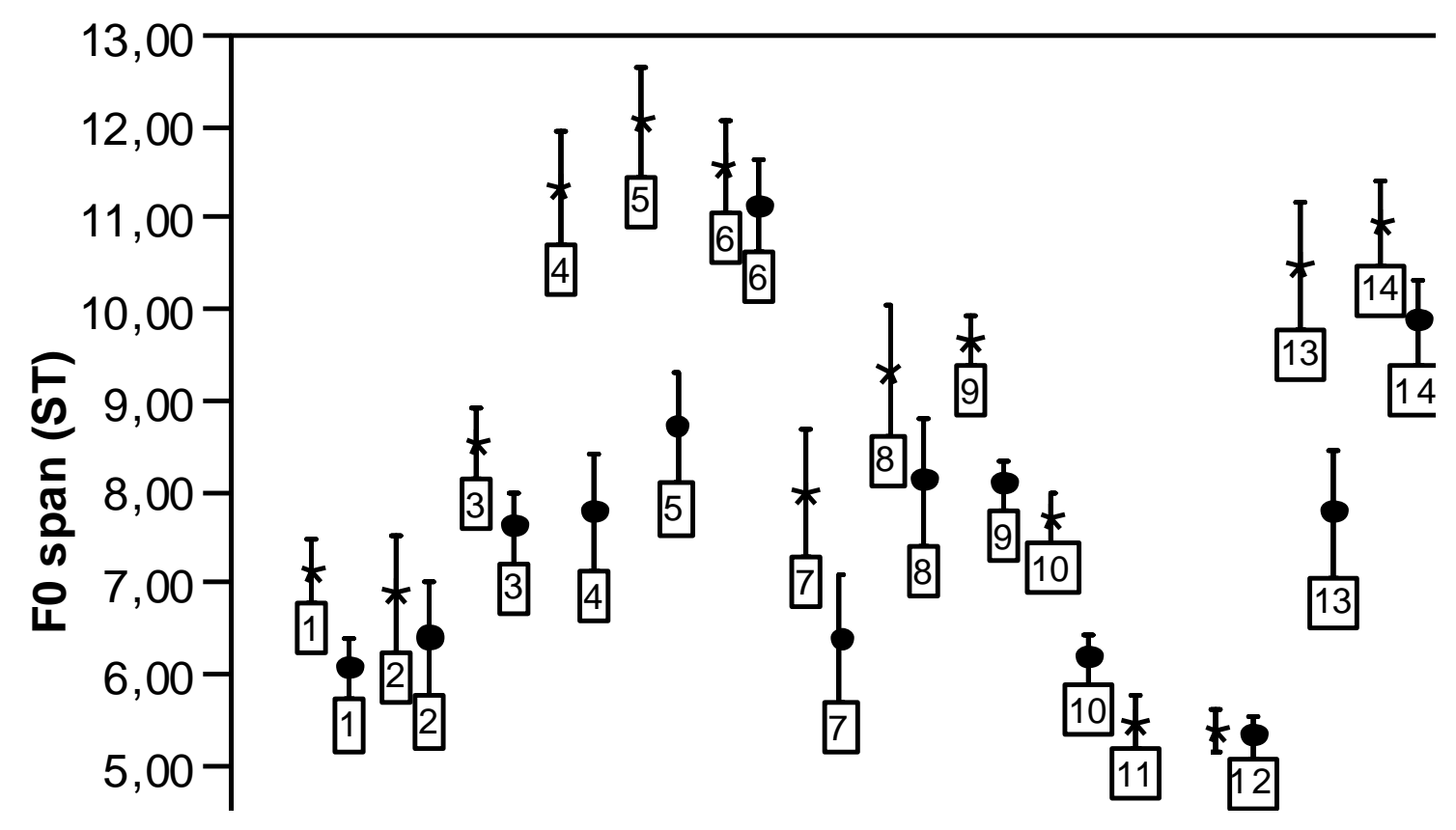


Figure 3b. Individual differences between Welsh and English in one of the level measures (F0 mean) in female bilinguals. Numbers on the graphs stand for the participant's number. Error bars $\pm 2 \mathrm{SE}$.

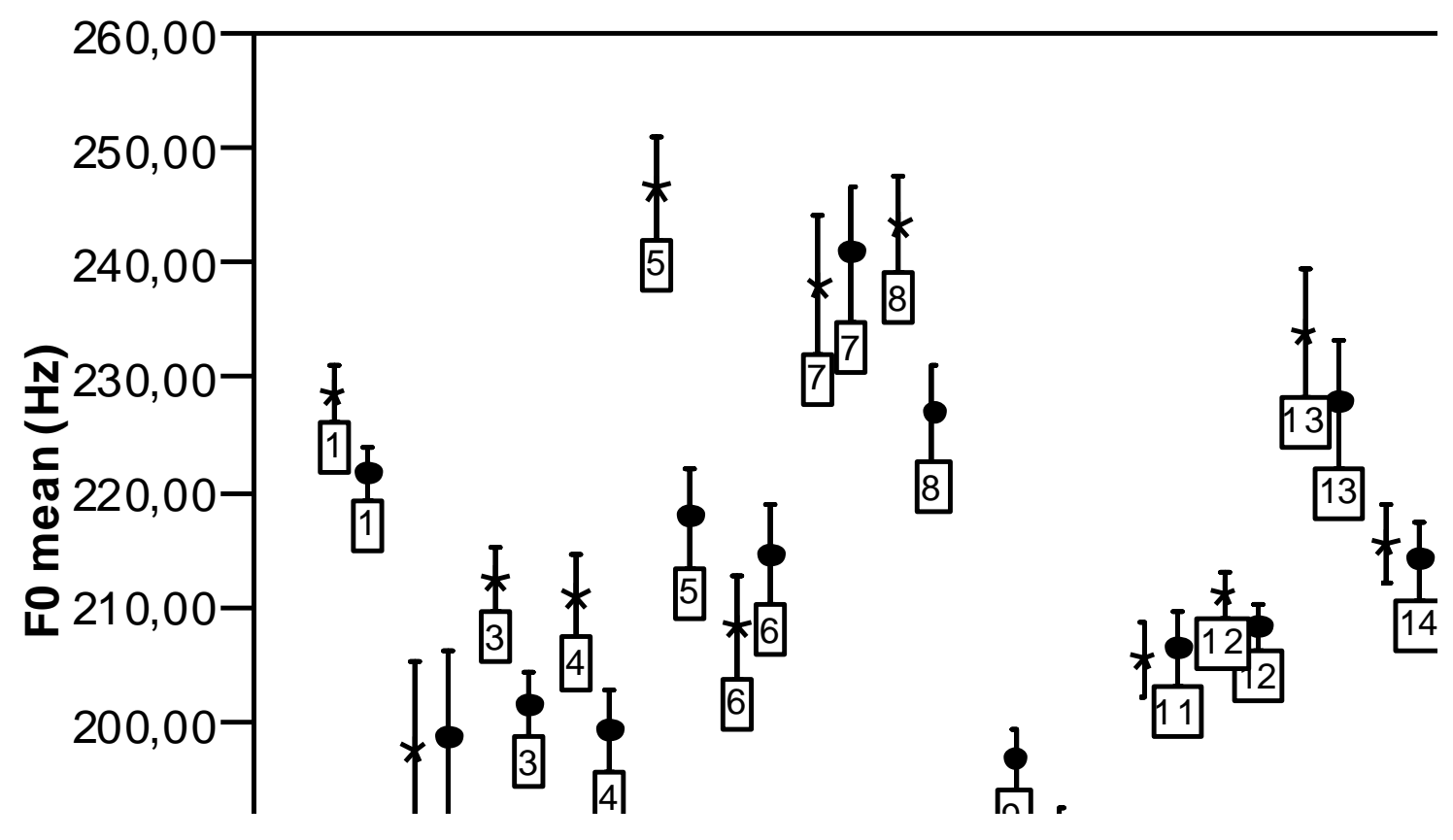


Figure 3c. Individual differences between Welsh and English in one of the level measures (F0 maxima) in female bilinguals. Numbers on the graphs stand for the participant's number. Error bars $\pm 2 \mathrm{SE}$.

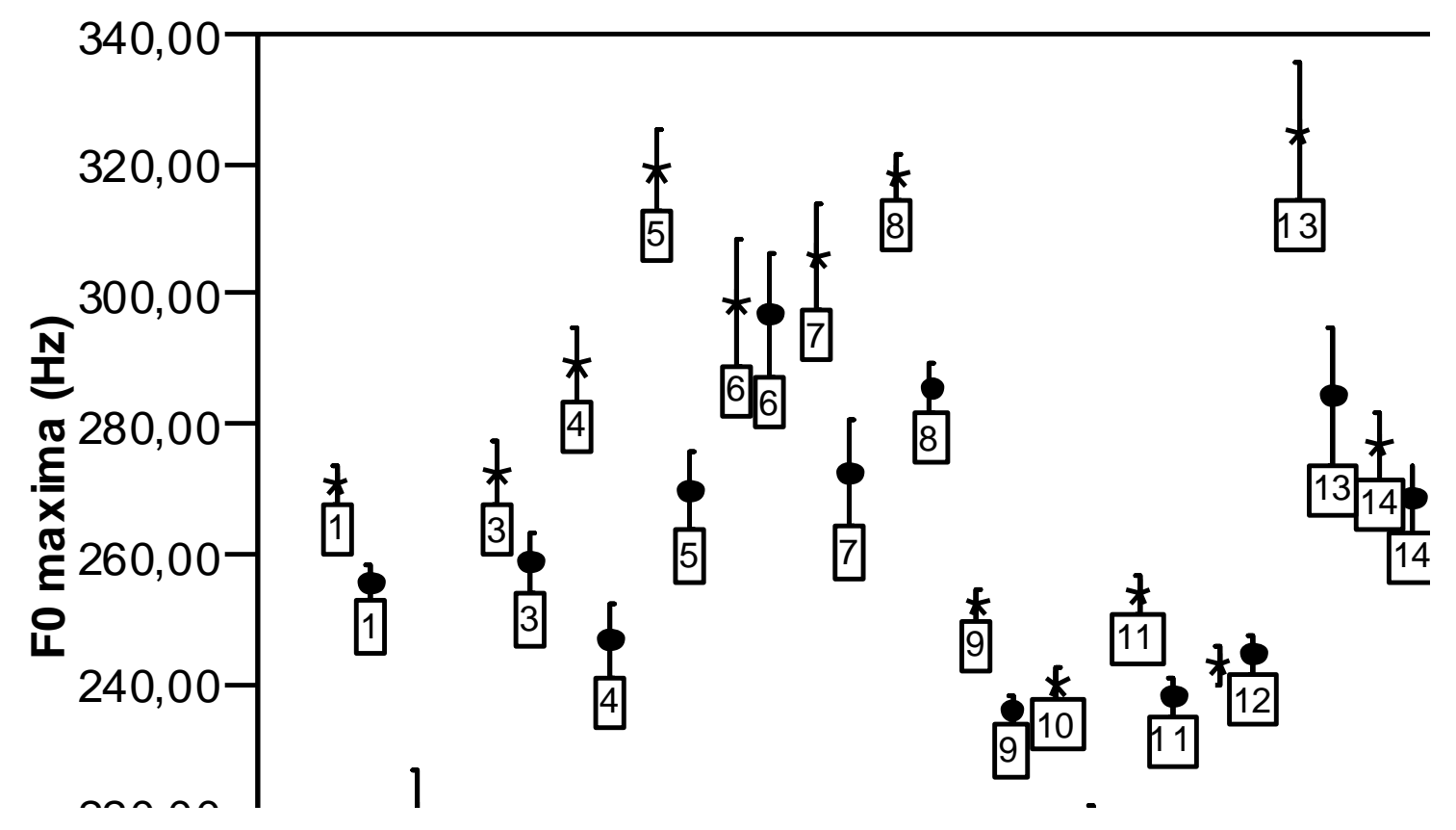


Figure 3d. Individual differences between Welsh and English in one of the level measures (F0 minima) in female bilinguals. Numbers on the graphs stand for the participant's number. Error bars \pm 2 SE.

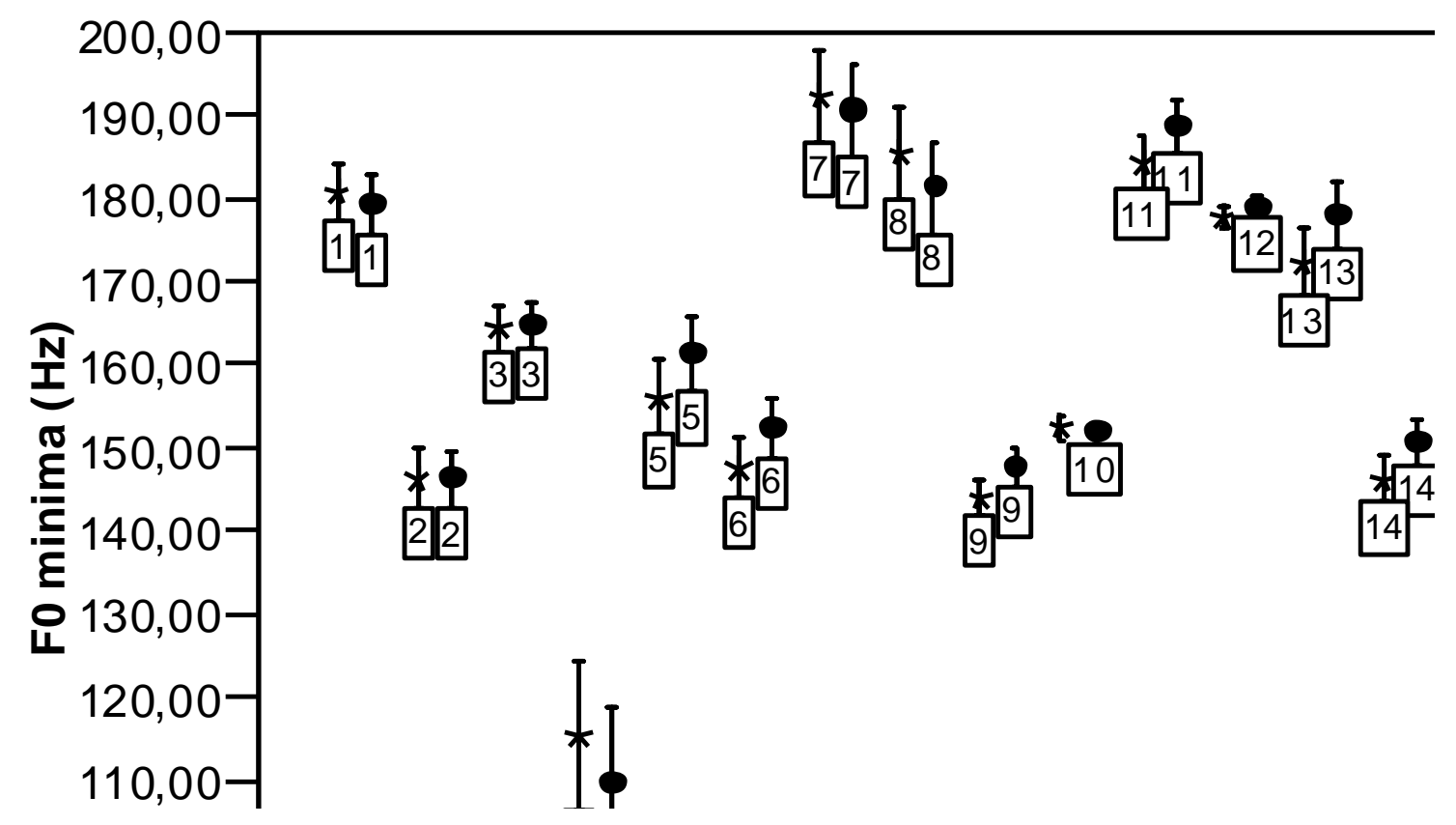


Figure 4a. Individual differences between Welsh and English in span in male bilinguals. Numbers on the graphs stand for the participant's number. Error bars $\pm 2 \mathrm{SE}$.

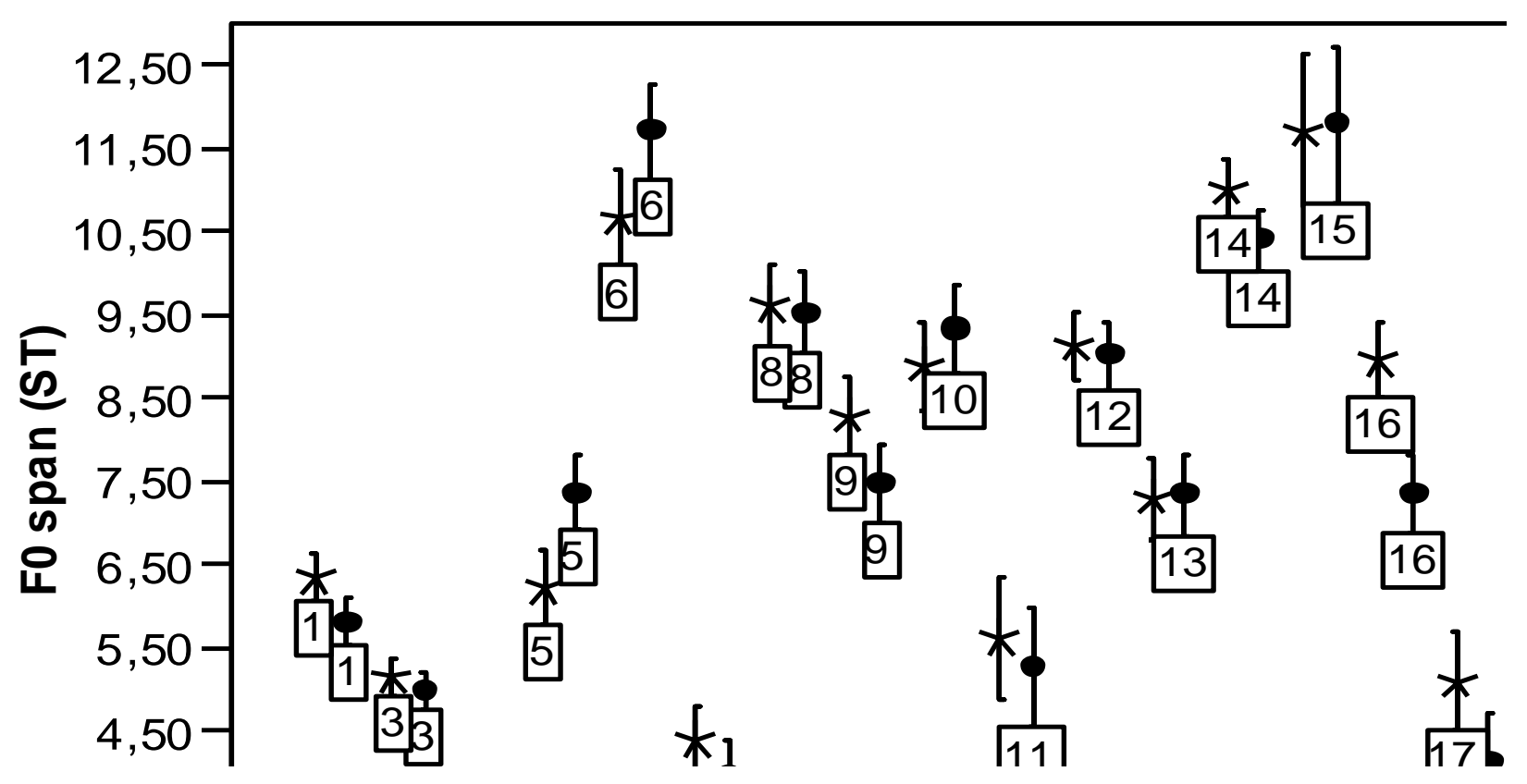


Figure 4b. Individual differences between Welsh and English in F0 mean in male bilinguals. Numbers on the graphs stand for the participant's number. Error bars $\pm 2 \mathrm{SE}$.

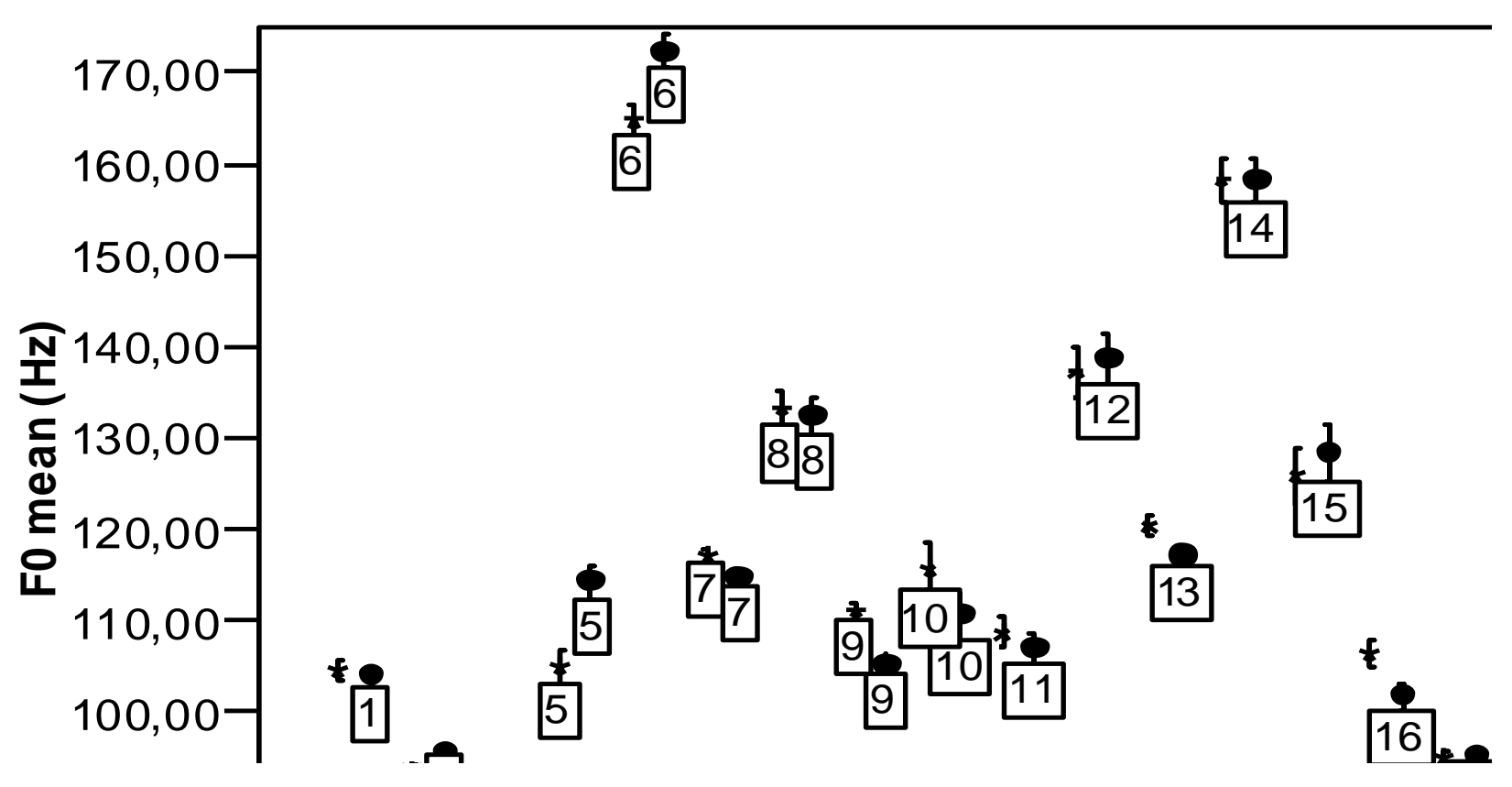


Figure 4c. Individual differences between Welsh and English in F0 maxima in male bilinguals. Numbers on the graphs stand for the participant's number. Error bars \pm 2 SE.

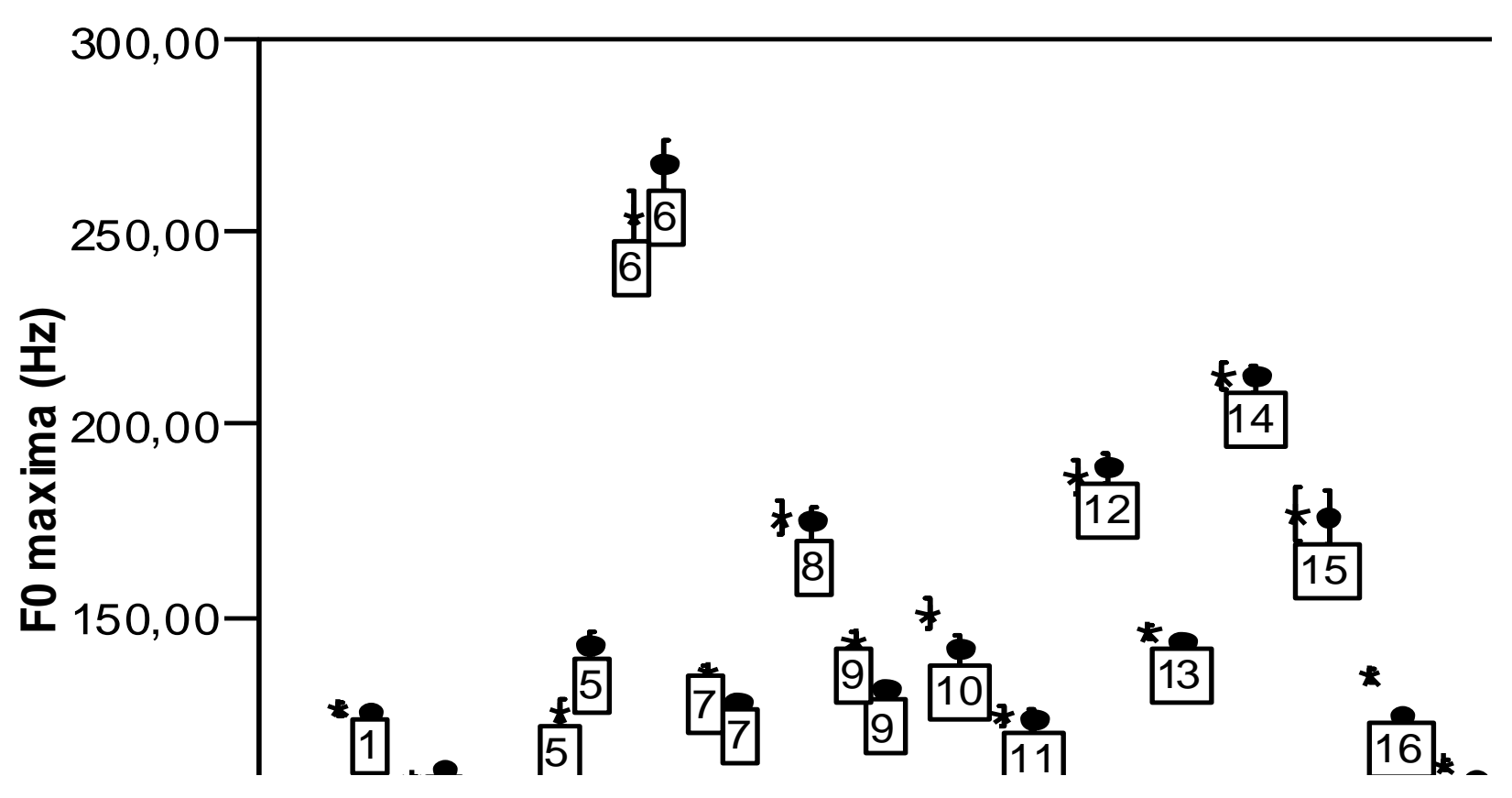


Figure 4d. Individual differences between Welsh and English in F0 minima in male bilinguals. Numbers on the graphs stand for the participant's number. Error bars \pm 2 SE.

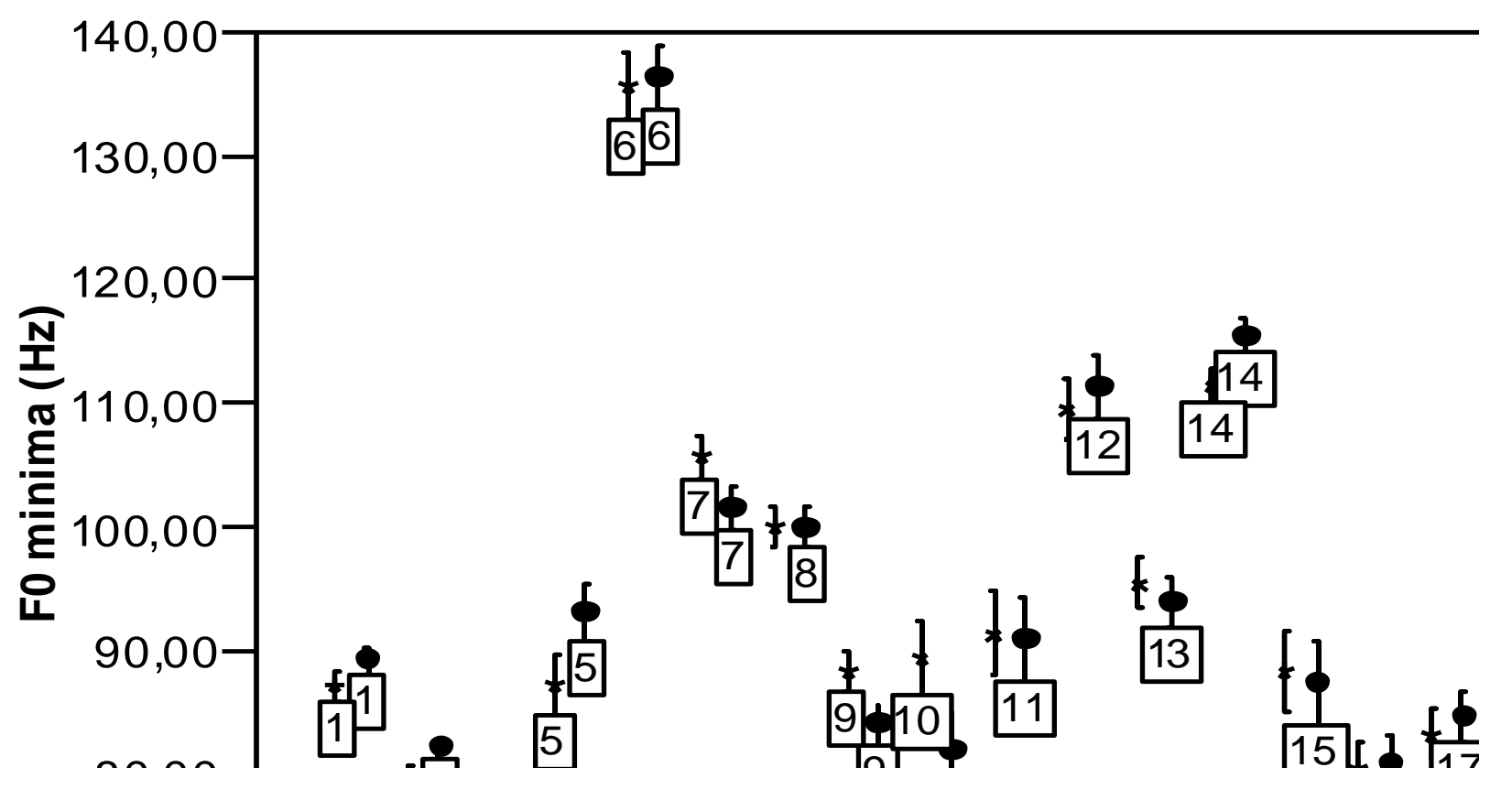




\begin{tabular}{|l|l|l|l|l|l|}
\hline Gender & Language & Span (ST) & F0max (Hz) & F0min (Hz) & F0mean (Hz) \\
\hline Males (N=16) & Welsh & $7.61(2.55)$ & $150.1(42)$ & $94.19(15)$ & $117.56(21.96)$ \\
\hline & English & $7.44(2.66)$ & $149.27(44.56)$ & $94.37(15.61)$ & $117.61(23.28)$ \\
\hline Females (N=14) & Welsh & $8.81(2.21)$ & $277.42(33)$ & $164.16(17.23)$ & $217.09(17.71)$ \\
\hline & English & $7.41(1.91)$ & $256.25(25.1)$ & $166.41(15.97)$ & $211.44(14.06)$ \\
\hline
\end{tabular}

Table 1. Means and standard deviations (in brackets) for FFR measures for bilingual speakers of Welsh and English. 


\begin{tabular}{|c|c|c|c|c|c|c|c|c|c|c|c|c|c|c|c|c|c|}
\hline \multicolumn{2}{|c|}{ Speaker } & \multicolumn{4}{|c|}{ F0 Span } & \multicolumn{4}{|c|}{ F0 max } & \multicolumn{4}{|c|}{ F0 mean } & \multicolumn{4}{|c|}{ F0 min } \\
\hline ID & Gender & $z$ & $p$ & $r$ & $\Delta$ & $z$ & $p$ & $r$ & $\Delta$ & $z$ & $p$ & $r$ & $\Delta$ & $z$ & $p$ & $r$ & $\Delta$ \\
\hline 1 & fem & -2.56 & .02 & .31 & $1.06 \mathrm{ST}, \mathrm{W}>\mathrm{E}$ & -4.371 & $<.0005$ & .52 & $1 \mathrm{ST}, \mathrm{W}>\mathrm{E}$ & -2.789 & .015 & .33 & $0.5 \mathrm{ST}, \mathrm{W}>\mathrm{E}$ & -.285 & .775 & .03 & $0.1 \mathrm{ST}, \mathrm{W}>\mathrm{E}$ \\
\hline 2 & fem & -.976 & .329 & .12 & $0.5 \mathrm{ST}, \mathrm{W}>\mathrm{E}$ & -1.129 & 1.0 & .14 & $0.6 S T, W>E$ & -.478 & .632 & .06 & $0.1 \mathrm{ST}, \mathrm{E}>\mathrm{W}$ & -.735 & .462 & .09 & $0, W=E$ \\
\hline 3 & fem & -2.139 & .064 & .26 & $0.92 \mathrm{ST}, \mathrm{W}>\mathrm{E}$ & -2.614 & .027 & .31 & $0.9 \mathrm{ST}, \mathrm{W}>\mathrm{E}$ & -3.482 & $<.0005$ & .42 & $0.9 \mathrm{ST}, \mathrm{W}>\mathrm{E}$ & -.417 & .677 & .05 & $0, W=E$ \\
\hline 4 & fem & -4.977 & $<.0005$ & .59 & $3.56 \mathrm{ST}, \mathrm{W}>\mathrm{E}$ & -5.332 & $<.0005$ & .64 & $2.7 \mathrm{ST}, \mathrm{W}>\mathrm{E}$ & -3.152 & .004 & .38 & $1 \mathrm{ST}, \mathrm{W}>\mathrm{E}$ & -.327 & .744 & .04 & $0.9 \mathrm{ST}, \mathrm{W}>\mathrm{E}$ \\
\hline 5 & fem & -4.575 & $<.0005$ & .55 & $3.36 \mathrm{ST}, \mathrm{W}>\mathrm{E}$ & -5.334 & $<.0005$ & .64 & 2.9ST, $W>E$ & -4.855 & $<.0005$ & .58 & 2.1ST, $W>E$ & -.893 & .372 & .11 & $0.6 \mathrm{ST}, \mathrm{E}>\mathrm{W}$ \\
\hline 6 & fem & -1.044 & .296 & .12 & $0.43 \mathrm{ST}, \mathrm{W}>\mathrm{E}$ & -.260 & .795 & .03 & $0.1 \mathrm{ST}, \mathrm{W}>\mathrm{E}$ & -1.349 & .608 & .16 & $0.5 \mathrm{ST}, \mathrm{E}>\mathrm{W}$ & -.648 & .517 & .08 & $0.6 \mathrm{ST}, \mathrm{E}>\mathrm{W}$ \\
\hline 7 & fem & -2.656 & .024 & .32 & $1.59 \mathrm{ST}, \mathrm{W}>\mathrm{E}$ & -3.605 & $<.0005$ & .43 & $2 \mathrm{ST}, \mathrm{W}>\mathrm{E}$ & -.833 & .81 & .1 & $0.2 \mathrm{ST}, \mathrm{E}>\mathrm{W}$ & -.726 & .468 & .09 & $0.2 \mathrm{ST}, \mathrm{W}>\mathrm{E}$ \\
\hline 8 & fem & -2.560 & .02 & .31 & $1.22 \mathrm{ST}, \mathrm{W}>\mathrm{E}$ & -5.392 & $<.0005$ & .64 & 1.9ST, $W>E$ & -3.307 & .003 & .4 & 1.2ST, $W>E$ & -.400 & .689 & .05 & $0.4 \mathrm{ST}, \mathrm{W}>\mathrm{E}$ \\
\hline 9 & fem & -4.633 & $<.0005$ & .55 & $1.58 \mathrm{ST}, \mathrm{W}>\mathrm{E}$ & -4.857 & $<.0005$ & .58 & 1.1ST, $W>E$ & -2.988 & .006 & .36 & $0.7 \mathrm{ST}, \mathrm{E}>\mathrm{W}$ & -1.813 & .070 & .22 & $0.4 \mathrm{ST}, \mathrm{E}>\mathrm{W}$ \\
\hline 10 & fem & -4.571 & $<.0005$ & .55 & $1.55 \mathrm{ST}, \mathrm{W}>\mathrm{E}$ & -5.684 & $<.0005$ & .68 & $1.6 \mathrm{ST}, \mathrm{W}>\mathrm{E}$ & -2.882 & .008 & .34 & $0.4 \mathrm{ST}, \mathrm{W}>\mathrm{E}$ & -.006 & .995 & .00 & $0.1 \mathrm{ST}, \mathrm{W}>\mathrm{E}$ \\
\hline 11 & fem & -4.070 & $<.0005$ & .49 & $1.5 \mathrm{ST}, \mathrm{W}>\mathrm{E}$ & -4.128 & $<.0005$ & .49 & 1.1ST, $W>E$ & -.369 & .712 & .04 & $0.1 S T, E>W$ & -2.230 & .052 & .27 & $0.4 \mathrm{ST}, \mathrm{E}>\mathrm{W}$ \\
\hline 12 & fem & -.024 & .981 & .00 & $0,05 W>E$ & -.815 & .415 & .1 & $0.1 S T, E>W$ & -.945 & .345 & .11 & $0.2 \mathrm{ST}, \mathrm{W}>\mathrm{E}$ & -1.454 & .584 & .17 & $0.1 \mathrm{ST}, \mathrm{E}>\mathrm{W}$ \\
\hline 13 & fem & -3.447 & .003 & .41 & 2.71ST.W>E & -3.207 & .004 & .38 & $2.3 \mathrm{ST}, \mathrm{W}>\mathrm{E}$ & -.978 & .328 & .12 & $0.5 \mathrm{ST}, \mathrm{W}>\mathrm{E}$ & -1.932 & .106 & .23 & $0.6 \mathrm{ST}, \mathrm{E}>\mathrm{W}$ \\
\hline 14 & fem & -2.486 & .052 & .3 & $1.09 \mathrm{ST}, \mathrm{W}>\mathrm{E}$ & -1.582 & .114 & .19 & $0.5 \mathrm{ST}, \mathrm{W}>\mathrm{E}$ & -.887 & .375 & .11 & $0.1 \mathrm{ST}, \mathrm{W}>\mathrm{E}$ & -1.794 & .073 & .21 & $0.5 \mathrm{ST}, \mathrm{E}>\mathrm{W}$ \\
\hline 1 & male & -1.474 & 0.56 & 0.18 & $0.54 \mathrm{ST}, \mathrm{W}>\mathrm{E}$ & -0.295 & 0.768 & 0.035 & $0.14 S T, W>E$ & -0.591 & 0.555 & 0.07 & OST, $W=E$ & -1.18 & 0.238 & 0.14 & $0.39 \mathrm{ST}, \mathrm{E}>\mathrm{W}$ \\
\hline 3 & male & -0.573 & 0.566 & 0.07 & $0.17 \mathrm{ST}, \mathrm{W}>\mathrm{E}$ & -1.865 & 0.124 & 0.22 & $0.32 \mathrm{ST}, \mathrm{E}>\mathrm{W}$ & -3.131 & 0.008 & 0.37 & $0.36 \mathrm{ST}, \mathrm{E}>\mathrm{W}$ & -2.594 & 0.027 & 0.31 & $0.43 \mathrm{ST}, \mathrm{E}>\mathrm{W}$ \\
\hline 4 & male & -0.761 & 0.447 & 0.09 & $0.11 \mathrm{ST}, \mathrm{E}>\mathrm{W}$ & -0.581 & 0.561 & 0.07 & $0.74 \mathrm{ST}, \mathrm{E}>\mathrm{W}$ & -0.645 & 0.519 & 0.078 & $0.2 \mathrm{ST}, \mathrm{E}>\mathrm{W}$ & -1.516 & 0.516 & 0.18 & $0.46 \mathrm{ST}, \mathrm{E}>\mathrm{W}$ \\
\hline 5 & male & -2.309 & 0.042 & 0.28 & $1.13 \mathrm{ST}, \mathrm{E}>\mathrm{W}$ & -4.185 & $<.0005$ & 0.5 & $2.33 \mathrm{ST}, \mathrm{E}>\mathrm{W}$ & -3.942 & $<.0005$ & 0.47 & $1.42 \mathrm{ST}, \mathrm{E}>\mathrm{W}$ & -2.171 & 0.03 & 0.26 & 1.15ST, $\mathrm{E}>\mathrm{W}$ \\
\hline
\end{tabular}




\begin{tabular}{|c|c|c|c|c|c|c|c|c|c|c|c|c|c|c|c|c|c|}
\hline 6 & male & -1.753 & 0.08 & 0.21 & 1.04ST, E>W & -2.089 & 0.111 & 0.25 & $0.86 \mathrm{ST}, \mathrm{E}>\mathrm{W}$ & -3.459 & 0.004 & 0.41 & $0.72 \mathrm{ST}, \mathrm{E}>\mathrm{W}$ & -0.59 & 0.555 & 0.07 & OST, W=E \\
\hline 7 & male & -1.016 & 0.31 & 0.12 & 3.96ST, $W>E$ & -4.319 & $<.0005$ & 0.52 & $1.05 \mathrm{ST}, \mathrm{W}>\mathrm{E}$ & -3.124 & 0.004 & 0.37 & $0.3 \mathrm{ST}, \mathrm{W}>\mathrm{E}$ & -4.082 & $<.0005$ & 0.49 & $0.67 \mathrm{ST}, \mathrm{W}>\mathrm{E}$ \\
\hline 8 & male & -0.197 & 0.844 & 0.02 & $0.08 \mathrm{ST}, \mathrm{W}>\mathrm{E}$ & -0.239 & 0.811 & 0.03 & $0.2 \mathrm{ST}, \mathrm{W}>\mathrm{E}$ & -0.18 & 0.857 & 0.02 & $0.13 \mathrm{ST}, \mathrm{W}>\mathrm{E}$ & -1.452 & 0.584 & 0.17 & OST, W=E \\
\hline 9 & male & -2.342 & 0.019 & 0.28 & $0.81, W>E$ & -4.326 & $<.0005$ & 0.52 & $1.64 \mathrm{ST}, \mathrm{W}>\mathrm{E}$ & -4.555 & $<.0005$ & 0.54 & $0.96 \mathrm{ST}, \mathrm{W}>\mathrm{E}$ & -2.78 & .01 & 0.33 & $0.8 \mathrm{ST}, \mathrm{W}>\mathrm{E}$ \\
\hline 10 & male & -0.426 & 0.67 & 0.05 & $0.42 \mathrm{ST}, \mathrm{E}>\mathrm{W}$ & -2.245 & 0.075 & 0.27 & 1.19ST, $W>E$ & -1.471 & 0.141 & 0.14 & $0.76 \mathrm{ST}, \mathrm{W}>\mathrm{E}$ & -2.515 & 0.048 & 0.3 & $1.42 \mathrm{ST}, \mathrm{W}>\mathrm{E}$ \\
\hline 11 & male & -0.157 & 0.875 & 0.02 & $0.36 \mathrm{ST}, \mathrm{W}>\mathrm{E}$ & -0.259 & 0.795 & 0.03 & $0.14 \mathrm{ST}, \mathrm{W}>\mathrm{E}$ & -1.13 & 1.0 & 0.13 & $0.38 \mathrm{ST}, \mathrm{W}>\mathrm{E}$ & -0.205 & 0.837 & 0.02 & OST, $W=E$ \\
\hline 12 & male & -0.803 & 0.422 & 0.1 & $0.12 \mathrm{ST}, \mathrm{W}>\mathrm{E}$ & -4.465 & 0.642 & 0.06 & $0.28 \mathrm{ST}, \mathrm{E}>\mathrm{W}$ & -1.292 & 0.588 & 0.15 & $0.2 \mathrm{ST}, \mathrm{E}>\mathrm{W}$ & -2.717 & 0.028 & 0.32 & $0.31 \mathrm{ST}, \mathrm{E}>\mathrm{W}$ \\
\hline 13 & male & -1.147 & 0.251 & 0.17 & $0.09 \mathrm{ST}, \mathrm{W}>\mathrm{E}$ & -1.762 & 0.078 & 0.21 & $0.36 \mathrm{ST}, \mathrm{W}>\mathrm{E}$ & -2.339 & 0.076 & 0.28 & $0.44 \mathrm{ST}, \mathrm{W}>\mathrm{E}$ & -0.189 & 0.85 & 0.02 & $0.18 \mathrm{ST}, \mathrm{W}>\mathrm{E}$ \\
\hline 14 & male & -1.54 & 0.372 & 0.184 & $0.42 \mathrm{ST}, \mathrm{W}>\mathrm{E}$ & -0.309 & 0.758 & 0.037 & OST, $W=E$ & -0.308 & 0.758 & 0.037 & OST, $W=E$ & -2.739 & 0.024 & 0.327 & $0.61 S T, E>W$ \\
\hline 15 & male & -0.639 & 0.523 & 0.08 & $0.1 S T, E>W$ & -0.606 & 0.544 & 0.072 & $0.1 \mathrm{ST}, \mathrm{W}>\mathrm{E}$ & -1.117 & 1.0 & 0.134 & $0.27, E>W$ & -0.385 & 0.7 & 0.05 & $0.2 \mathrm{ST}, \mathrm{W}>\mathrm{E}$ \\
\hline 16 & male & -3.063 & 0.004 & 0.37 & $1.6 \mathrm{ST}, \mathrm{W}>\mathrm{E}$ & -4.678 & $<.0005$ & 0.56 & $1.47 \mathrm{ST}, \mathrm{W}>\mathrm{E}$ & -3.113 & 0.006 & 0.37 & $0.67 \mathrm{ST}, \mathrm{W}>\mathrm{E}$ & -1.335 & 0.182 & 0.15 & OST, W=E \\
\hline 17 & male & -3.103 & 0.006 & 0.37 & $0.98 \mathrm{ST}, \mathrm{W}>\mathrm{E}$ & -3.126 & 0.008 & 0.37 & $0.79 S T, W>E$ & -0.019 & 0.985 & 0.00 & OST, W=E & -2.407 & 0.032 & 0.29 & $0.42 \mathrm{ST}, \mathrm{E}>\mathrm{W}$ \\
\hline
\end{tabular}

Table 2. Significance of cross-linguistic differences in FFR between Welsh and English in bilinguals' speech. 


\begin{tabular}{|c|c|c|c|c|c|c|c|c|c|c|c|c|c|c|c|c|}
\hline Speaker & 1 & 3 & 4 & 5 & 6 & 7 & 8 & 9 & 10 & 11 & 12 & 13 & 14 & 15 & 16 & 17 \\
\hline Language of exposure between 0 and 4 years & $\mathrm{E}$ & most. E & $\mathrm{W}$ & most. E & $\mathrm{W} / \mathrm{E}$ & most. W & $\mathrm{W}$ & $\mathrm{W}$ & $\mathrm{W}$ & $\mathrm{W}$ & $\mathrm{W} / \mathrm{E}$ & most. W & most. W & $\mathrm{W} / \mathrm{E}$ & $\mathrm{W}$ & $\mathrm{W}$ \\
\hline Language of exposure between 4 and 9 years & most. E & most. E & most. W & $\mathrm{W} / \mathrm{E}$ & $\mathrm{W} / \mathrm{E}$ & most. W & $\mathrm{W}$ & most. W & most. W & most. W & $\mathrm{W} / \mathrm{E}$ & most. W & most. W & $\mathrm{W} / \mathrm{E}$ & $\mathrm{W}$ & $\mathrm{W}$ \\
\hline Language of exposure between 9 and 15 years & most. E & most. E & most. W & most. W & $\mathrm{W} / \mathrm{E}$ & $\mathrm{W} / \mathrm{E}$ & most. W & most. W & most. W & most. W & $\mathrm{W} / \mathrm{E}$ & most. W & most. W & W/E & $\mathrm{W}$ & $\mathrm{W}$ \\
\hline Language used to talk to father & $\mathrm{E}$ & E & $\mathrm{W}$ & $\mathrm{E}$ & E & $\mathrm{W}$ & $\mathrm{W}$ & $\mathrm{W}$ & $\mathrm{W}$ & $\mathrm{W}$ & most. E & $\mathrm{W}$ & $\mathrm{W} / \mathrm{E}$ & most. E & $\mathrm{W}$ & $\mathrm{W}$ \\
\hline Language used to talk to mother & E & most. E & $\mathrm{W}$ & most. E & E & $\mathrm{W}$ & W & $\mathrm{W}$ & $\mathrm{W}$ & $\mathrm{W}$ & $\mathrm{W} / \mathrm{E}$ & $\mathrm{W}$ & most. W & most. W & $\mathrm{W}$ & $\mathrm{W}$ \\
\hline Language used between parents & E & E & $\mathrm{W}$ & $\mathrm{E}$ & E & $\mathrm{W}$ & W & $\mathrm{W}$ & $\mathrm{W}$ & $\mathrm{W}$ & most. E & most. E & $\mathrm{W} / \mathrm{E}$ & $\mathrm{W} / \mathrm{E}$ & $\mathrm{W}$ & W \\
\hline
\end{tabular}

Table 3. Language use by male Welsh-English bilinguals ("E" - English; "most. E" - mostly English; "most. W" - mostly Welsh; "W" - Welsh; "E/W" - English and Welsh without preference). 


\begin{tabular}{|c|c|c|c|c|c|c|c|c|c|c|c|c|c|c|}
\hline Speaker & 1 & 2 & 3 & 4 & 5 & 6 & 7 & 8 & 9 & 10 & 11 & 12 & 13 & 14 \\
\hline Language of exposure between 0 and 4 years & W & $\mathrm{W}$ & W & $\mathrm{W}$ & $\mathrm{W} / \mathrm{E}$ & $\mathrm{W}$ & $\mathrm{W}$ & W & $\mathrm{W}$ & W & W & $\mathrm{W}$ & $\mathrm{W} / \mathrm{E}$ & W \\
\hline Language of exposure between 4 and 9 years & W & W & W & W & E & $\mathrm{W} / \mathrm{E}$ & W & $\mathrm{W} / \mathrm{E}$ & $\mathrm{W} / \mathrm{E}$ & W & $\mathrm{W}$ & W & $\mathrm{W}$ & W \\
\hline Language of exposure between 9 and 15 years & $\mathrm{W}$ & $\mathrm{W}$ & W & $\mathrm{W}$ & $\mathrm{E}$ & $\mathrm{W} / \mathrm{E}$ & $\mathrm{W}$ & W/E & $\mathrm{W} / \mathrm{E}$ & $\mathrm{W}$ & $\mathrm{E}$ & W & $\mathrm{W}$ & W \\
\hline Language used to talk to father & $\mathrm{W}$ & W & $\mathrm{W} / \mathrm{E}$ & $\mathrm{E}$ & $\mathrm{E}$ & $\mathrm{E}$ & $\mathrm{E}$ & $\mathrm{E}$ & $\mathrm{E}$ & $\mathrm{W}$ & $\mathrm{E}$ & $\mathrm{E}$ & E & W \\
\hline Language used to talk to mother & W & W & $\mathrm{W} / \mathrm{E}$ & W & W & $\mathrm{W}$ & $\mathrm{W}$ & $\mathrm{W}$ & W & $\mathrm{W}$ & $\mathrm{W} / \mathrm{E}$ & $\mathrm{W}$ & $\mathrm{W}$ & $\mathrm{W}$ \\
\hline Language used between parents & W & W & W/E & W/E & $\mathrm{W} / \mathrm{E}$ & W/E & W/E & $\mathrm{W} / \mathrm{E}$ & $\mathrm{W} / \mathrm{E}$ & W & W/E & $\mathrm{W} / \mathrm{E}$ & $\mathrm{W} / \mathrm{E}$ & W \\
\hline
\end{tabular}

Table 4. Language use by female Welsh-English bilinguals ("E" - English; "most. E" - mostly English; "most. W" - mostly Welsh; "W" - Welsh; "E/W" - English and Welsh without preference). 